\title{
Soluble products of connected subgroups
}

\section{Pilar Gállego, Peter Hauck and M. Dolores Pérez-Ramos}

\author{
Dedicated to James C. Beidleman on his 70th birthday
}

\begin{abstract}
The main result in the paper states the following: For a finite group $G=A B$, which is the product of the soluble subgroups $A$ and $B$, if $\langle a, b\rangle$ is a metanilpotent group for all $a \in A$ and $b \in B$, then the factor groups $\langle a, b\rangle F(G) / F(G)$ are nilpotent, $F(G)$ denoting the Fitting subgroup of $G$. A particular generalization of this result and some consequences are also obtained. For instance, such a group $G$ is proved to be soluble of nilpotent length at most $l+1$, assuming that the factors $A$ and $B$ have nilpotent length at most $l$. Also for any finite soluble group $G$ and $k \geq 1$, an element $g \in G$ is contained in the preimage of the hypercenter of $G / F_{k-1}(G)$, where $F_{k-1}(G)$ denotes the $(k-1)$ th term of the Fitting series of $G$, if and only if the subgroups $\langle g, h\rangle$ have nilpotent length at most $k$ for all $h \in G$.
\end{abstract}

\section{Introduction}

The study of factorized groups whose factors are linked by some particular property has received considerable interest recently. The focus in this paper is on a connection property introduced by Carocca [5] (based on a remark of Maier in [14]):

Let $\mathcal{L}$ be a non-empty class of groups. Subgroups $A$ and $B$ of a group $G$ are $\mathcal{L}$-connected if $\langle a, b\rangle \in \mathcal{L}$ for all $a \in A$ and $b \in B$. A group $G=A B$ is an $\mathcal{L}$-connected product of $A$ and $B$ if $A$ and $B$ are $\mathcal{L}$-connected. Of course, the special case $A=B=G$ has been dealt with before; there are

2000 Mathematics Subject Classification: 20D10, $20 \mathrm{D} 40$.

Keywords: Finite groups, soluble groups, 2-generated subgroups, product of subgroups, metanilpotent groups, Fitting series. 
numerous results considering the question when for a group $G$ containment of all 2-generated subgroups in $\mathcal{L}$ implies that $G$ is contained in $\mathcal{L}$. For finite groups, the most famous result in this direction is a consequence of Thompson's classification of minimal simple groups [15]:

A finite group is soluble if all of its 2-generated subgroups are soluble.

A nice elementary proof of this theorem has been given by Flavell [9]. As a further example we mention the work of Carter, Fischer and Hawkes [7] where results of the same type are obtained for various important subclasses of finite soluble groups.

We note also that results of this type follow from the theory of varieties of groups, and for finite groups in particular from studying finite varieties introduced by Brandl in [4].

For general $\mathcal{L}$-connected products, up to now only the cases $\mathcal{L}=\mathcal{S}$, the class of finite soluble groups, and $\mathcal{L}=\mathcal{N}$, the class of finite nilpotent groups, have been studied.

Carocca [6] showed that $\mathcal{S}$-connected products of soluble groups are soluble. Structure and properties of $\mathcal{N}$-connected products are understood very well (cf. Ballester-Bolinches, Pedraza-Aguilera [2] and Hauck, Martínez-Pastor, Pérez-Ramos [12]). For instance, in an $\mathcal{N}$-connected product $G=A B$, $A$ and $B$ are subnormal subgroups and the nilpotent residual $A^{\mathcal{N}}$ of $A$ is centralized by $B$ (and vice versa). Also the product $G=A B$ is $\mathcal{N}$-connected if and only if $G$ modulo its hypercenter is a direct product of the images of $A$ and $B$. A further study of the behaviour of $\mathcal{N}$-connected products of groups, in relation to certain inheritance properties between the factors and the whole group, was carried out by Beidleman and Heineken [3].

In this paper we consider mainly the case $\mathcal{L}=\mathcal{N}^{2}$, the class of finite metanilpotent groups. It is obvious that $G=A B$ is an $\mathcal{N}^{2}$-connected product of $A$ and $B$ if $G / F(G), F(G)$ denoting the Fitting subgroup of $G$, is an $\mathcal{N}$-connected product of $A F(G) / F(G)$ and $B F(G) / F(G)$. The main theorem of this paper says that for soluble groups the converse holds, too. Thus, the structure of soluble $\mathcal{N}^{2}$-connected products is reduced to the structure of $\mathcal{N}$-connected products which is quite transparent by the results mentioned above.

It is an open question whether a corresponding statement is true if $\mathcal{N}^{2}$ is replaced by $\mathcal{N}^{k}$, the class of finite soluble groups of nilpotent length at most $k$, for $k \geq 3$. However, under certain conditions on $A$ and $B$ such a generalization can be obtained which has already interesting implications. For instance, let $k \geq 1$ and let $g$ be an element of a finite soluble group $G$; then $\langle g, h\rangle \in \mathcal{N}^{k}$ for all $h \in G$ if and only if $g$ is contained in the preimage of the hypercenter of $G / F_{k-1}(G)$, where $F_{k-1}(G)$ denotes the $(k-1)$ th term of the Fitting series of $G$. 


\section{Notation and preliminary results}

All groups considered in this paper are assumed to be finite.

We shall adhere to the notation used in [8] and we refer also to that book for the basic results on classes of groups. In particular, $\mathbf{P}$ denotes the set of all prime numbers and $\sigma(G)$ the set of all primes dividing the order of the group $G$. Also $\mathcal{A}$ denotes the class of all abelian groups.

We now gather some results on products of groups and on $\mathcal{N}$-connected products which will be needed in the paper.

Lemma 1 Let the group $G=A B$ be the product of two subgroups $A$ and $B$. Then:

1. ([1, Lemma 1.3.2]) If $A, B, G$ are $D_{\pi}$-groups for a set $\pi$ of primes, then there exist Hall $\pi$-subgroups $A_{0}$ of $A$ and $B_{0}$ of $B$ such that $A_{0} B_{0}$ is a Hall $\pi$-subgroup of $G$.

2. ([1, p. 3, Lemma 1.1.4(i)]) For a subgroup $S$ of $G$, the factorizer $X(S)$ of $S$ in $G=A B$ satisfies $S \leq X(S)=(A \cap X(S))(B \cap X(S))$. If in addition $S$ is normal in $G$, then $X(S)=A S \cap B S$.

3. ([13, Theorem 4.4.1]) If $A$ and $B$ are subnormal subgroups of $G$, then $G^{\mathcal{N}}=A^{\mathcal{N}} B^{\mathcal{N}}$.

Lemma 2 ([12, Proposition 1 (2), (8), Proposition 4]) Let the group $G=$ $A B$ be an $\mathcal{N}$-connected product of the subgroups $A$ and $B$. Then:

1. $A$ and $B$ are subnormal in $G$.

2. $A \cap B \leq Z_{\infty}(G) \leq F(G)$.

3. $F(G)=(F(G) \cap A)(F(G) \cap B)$.

Lemma 3 If two elements $x, y$ of a group $G$ have coprime orders, then $\langle x, y\rangle^{\mathcal{N}}=[\langle x\rangle,\langle y\rangle]$.

Lemma 4 Let the group $G=A B$ be the product of the subgroups $A$ and $B$. If $F(G)=(F(G) \cap A)(F(G) \cap B)$, then

$$
O_{s}(G)=\left(O_{s}(G) \cap A\right)\left(O_{s}(G) \cap B\right),
$$

for any prime $s$. If in addition $F_{2}(G)=\left(F_{2}(G) \cap A\right)\left(F_{2}(G) \cap B\right)$, then

$$
O_{s}:=O_{s}(G \bmod F(G))=\left(O_{s} \cap A\right)\left(O_{s} \cap B\right),
$$

for any primes. 
Proof. The first part is easily proved. From this part we deduce in order to complete the proof that

$$
\begin{aligned}
O_{s} & =\left(O_{s} \cap A F(G)\right)\left(O_{s} \cap B F(G)\right)=\left(O_{s} \cap A\right) F(G)\left(O_{s} \cap B\right)= \\
& =\left(O_{s} \cap A\right)(F(G) \cap A)(F(G) \cap B)\left(O_{s} \cap B\right)=\left(O_{s} \cap A\right)\left(O_{s} \cap B\right)
\end{aligned}
$$

and we are done.

Lemma 5 If the group $G=A B$ is an $\mathcal{L}$-connected product, then $G=A^{x} B^{y}$ is an $\mathcal{L}$-connected product, for any pair of elements $x, y \in G$.

Proof . Let $x, y \in G$. Since $G=A B$ it is known by [1, Lemma 1.3.1] that $G=A^{x} B^{y}$. Moreover, there exists an element $z$ of $G$ such that $A^{x}=A^{z}$ and $B^{y}=B^{z}$. The result follows now by a straightforward argument.

Lemma 6 Let the finite group $G=A B$ be the product of the subgroups $A$ and $B$. Then the following statements are pairwise equivalent:

(i) $A$ and $B$ are $\mathcal{N}$-connected.

(ii) For every pair of primes $p$ and $q$ such that $p \neq q,\left[A_{p}, B_{q}\right]=1$ for all $A_{p} \in \operatorname{Syl}_{p}(A)$ and all $B_{q} \in \operatorname{Syl}_{q}(B)$.

(iii) $\left(\right.$ a) $\left[A^{\mathcal{N}}, B\right]=1,\left[B^{\mathcal{N}}, A\right]=1$;

(b) for every pair of primes $p$ and $q$ such that $p \neq q$, there exist $A_{p} \in$ $\operatorname{Syl}_{p}(A)$ and $B_{q} \in \operatorname{Syl}_{q}(B)$ such that $\left[A_{p}, B_{q}\right]=1$.

Proof. (i) implies (ii). This is clear.

(ii) implies (iii). Let $q$ be a prime number and $B_{q} \in \operatorname{Syl}_{q}(B)$. From (ii) it follows that $\left[A^{\mathcal{N}}, B_{q}\right] \leq\left[O^{q}(A), B_{q}\right]=1$. Consequently $\left[A^{\mathcal{N}}, B\right]=1$. The second part is clear.

(iii) implies $(i)$. We notice that for every prime $p$, if $P \in \operatorname{Syl}_{p}(A)$, then $A^{\mathcal{N}} P$ is normal in $A$ and so $\operatorname{Syl}_{p}(A)=\left\{P^{t} \mid t \in A^{\mathcal{N}}\right\}$. Analogously, if $Q \in \operatorname{Syl}_{p}(B)$, then $\operatorname{Syl}_{p}(B)=\left\{Q^{t} \mid t \in B^{\mathcal{N}}\right\}$. Then $(i i)$ is easily deduced from $($ iii $)$. On the other hand, for every prime $p$, we recall that there exist $X_{p} \in \operatorname{Syl}_{p}(A)$ and $Y_{p} \in \operatorname{Syl}_{p}(B)$ such that $X_{p} Y_{p} \in \operatorname{Syl}_{p}(G)$. Then it can be also proved that every Sylow $p$-subgroup of $A$ permutes with every Sylow $p$-subgroup of $B$.

Now, let $a \in A, b \in B$ and let us consider $\langle a\rangle=\times_{p \in \mathbf{P}}\langle a\rangle_{p},\langle b\rangle=$ $\times_{p \in \mathbf{P}}\langle b\rangle_{p}$. It is clear that $\langle a, b\rangle=\times_{p \in \mathbf{P}}\left\langle\langle a\rangle_{p},\langle b\rangle_{p}\right\rangle$ is nilpotent and so $A$ and $B$ are $\mathcal{N}$-connected. 
Lemma 7 If $N$ is a subgroup of a group $G$ normalized by an element $g \in G$, then $[N,\langle g\rangle]=[N, g]$.

In addition, if $N$ is abelian, the map $N \longrightarrow[N, g]$, which sends each $n \in N$ to $[n, g] \in[N, g]$, is an epimorphism of groups with kernel $C_{N}(g)$. In particular, $N / C_{N}(g) \cong[N, g]=\{[n, g] \mid n \in N\}$.

The above-mentioned results will be used freely throughout the paper, usually without further reference.

Lemma 8 Let $\mathcal{F}$ be a formation of soluble groups. Let $G$ be a group, $N$ a subgroup of $G$ and $\alpha, \beta \in G$. We say that $N, \alpha, \beta$ satisfy Condition (*) provided that

$N$ is normalized by $\langle\alpha, \beta\rangle$,

$N$ is an abelian p-group for some prime $p$,

$O_{p}\left(\langle\alpha, \beta\rangle^{\mathcal{F}}\right) \leq N$ and

$\left\langle\alpha^{n}, \beta^{m}\right\rangle \in \mathcal{N} \mathcal{F}$ for all $n, m \in N$.

(It is clear that the last part of $(*)$ holds if $\left\langle\alpha, \beta^{n}\right\rangle \in \mathcal{N} \mathcal{F}$ for all $n \in N$.)

Assume that $N, \alpha, \beta$ satisfy Condition (*). Set

$$
\begin{gathered}
T=N\langle\alpha, \beta\rangle, C=C_{N}\left(\langle\alpha, \beta\rangle^{\mathcal{F}}\right), \quad R=\left[N,\langle\alpha, \beta\rangle^{\mathcal{F}}\right], \\
N_{1}=\{n \in N \mid\langle n \alpha, \beta\rangle \in \mathcal{N} \mathcal{F}\}, \quad N_{2}=\{n \in N \mid\langle\alpha, n \beta\rangle \in \mathcal{N} \mathcal{F}\} .
\end{gathered}
$$

Then:

1. $N=C \times R$ and $C\left\langle\alpha^{n}, \beta^{m}\right\rangle$ is an $\mathcal{N} \mathcal{F}$-projector of $T$ for all $n, m \in N$. (Notice that $\mathcal{N} \mathcal{F}$ is a saturated formation for any formation $\mathcal{F}$.)

2. $R=C_{R}(\alpha) \times C_{R}(\beta)$.

3. $N_{1}=C[N,\langle\alpha\rangle]=C[R, \alpha]$ and $N_{2}=C[N,\langle\beta\rangle]=C[R, \beta]$.

4. If $\mu \in N_{1}$, then $N, \mu \alpha, \beta$ satisfy Condition (*).

5. If $L_{1} \leq N_{1}, L_{2} \leq N_{2}$ and $N=L_{1} L_{2}$, then $C L_{1}=N_{1}, C L_{2}=N_{2}$.

6. If in addition $\mathcal{A} \subseteq \mathcal{F}$, then

$N=N_{1}$ if and only if $N=C$ if and only if $\beta$ normalizes $N_{1}$.

Proof. Note that $T=N\langle\alpha, \beta\rangle$ is a soluble group and that $C=C_{N}\left(\langle\alpha, \beta\rangle^{\mathcal{F}}\right)$ and $R=\left[N,\langle\alpha, \beta\rangle^{\mathcal{F}}\right]$ are normal subgroups of $T$.

1. Let $n, m \in N$. Notice that we have $N\left\langle\alpha^{n}, \beta^{m}\right\rangle=N\langle\alpha, \beta\rangle=T$, which implies that $N\left\langle\alpha^{n}, \beta^{m}\right\rangle^{\mathcal{F}}=N\langle\alpha, \beta\rangle^{\mathcal{F}}$. Since $\langle\alpha, \beta\rangle \in \mathcal{N} \mathcal{F}$ and $O_{p}\left(\langle\alpha, \beta\rangle^{\mathcal{F}}\right) \leq$ $N$, it follows that $N\langle\alpha, \beta\rangle^{\mathcal{F}}=N O_{p^{\prime}}\left(\langle\alpha, \beta\rangle^{\mathcal{F}}\right)$. Thus $O_{p}\left(\left\langle\alpha^{n}, \beta^{m}\right\rangle^{\mathcal{F}}\right) \leq N$ and consequently, $N O_{p^{\prime}}\left(\left\langle\alpha^{n}, \beta^{m}\right\rangle^{\mathcal{F}}\right)=N\left\langle\alpha^{n}, \beta^{m}\right\rangle^{\mathcal{F}}=N\langle\alpha, \beta\rangle^{\mathcal{F}}$. Since $N$ is 
abelian, we conclude that $C=C_{N}\left(\langle\alpha, \beta\rangle^{\mathcal{F}}\right)=C_{N}\left(O_{p^{\prime}}\left(\left\langle\alpha^{n}, \beta^{m}\right\rangle^{\mathcal{F}}\right)\right)$. Moreover, $R=\left[N, N\langle\alpha, \beta\rangle^{\mathcal{F}}\right]=\left[N, O_{p^{\prime}}\left(\left\langle\alpha^{n}, \beta^{m}\right\rangle^{\mathcal{F}}\right)\right]$. Therefore, by coprime action it follows that $N=R \times C$ and that $R=\left[R, O_{p^{\prime}}\left(\left\langle\alpha^{n}, \beta^{m}\right\rangle^{\mathcal{F}}\right)\right]$.

We claim that $C\left\langle\alpha^{n}, \beta^{m}\right\rangle$ is a complement of $R$ in $T$. We have $T=$ $N\left\langle\alpha^{n}, \beta^{m}\right\rangle=R C\left\langle\alpha^{n}, \beta^{m}\right\rangle$. Observe that $\left[N \cap\left\langle\alpha^{n}, \beta^{m}\right\rangle, O_{p^{\prime}}\left(\left\langle\alpha^{n}, \beta^{m}\right\rangle^{\mathcal{F}}\right)\right] \leq$ $N \cap O_{p^{\prime}}\left(\left\langle\alpha^{n}, \beta^{m}\right\rangle^{\mathcal{F}}\right)=1$. Hence $N \cap\left\langle\alpha^{n}, \beta^{m}\right\rangle \leq C$. Thus, $R \cap C\left\langle\alpha^{n}, \beta^{m}\right\rangle=$ $R \cap N \cap C\left\langle\alpha^{n}, \beta^{m}\right\rangle=R \cap C\left(N \cap\left\langle\alpha^{n}, \beta^{m}\right\rangle\right)=R \cap C=1$. This proves that $C\left\langle\alpha^{n}, \beta^{m}\right\rangle$ is a complement of $R$ in $T$.

Since $C\left\langle\alpha^{n}, \beta^{m}\right\rangle^{\mathcal{F}} \in \mathcal{N}$, it is clear that $T^{\mathcal{N F}} \leq R$. In particular, $T^{\mathcal{N} \mathcal{F}}$ is abelian and by [8, Theorem IV.5.18], the complements of $T^{\mathcal{N} \mathcal{F}}$ in $T$ are precisely the $\mathcal{N} \mathcal{F}$-projectors of $T$. Thus, to complete the proof of Part 1 it is sufficient to show that $T^{\mathcal{N} \mathcal{F}}=R$. Since $R=\left[R, N\langle\alpha, \beta\rangle^{\mathcal{F}}\right]=\left[R, T^{\mathcal{F}}\right]$, then $R \leq T^{\mathcal{F}}$. Furthermore $R \leq\left(T^{\mathcal{F}}\right)^{\mathcal{N}}=T^{\mathcal{N} \mathcal{F}} \leq R$ and we are done.

2. In order to show that $R=C_{R}(\alpha) C_{R}(\beta)$, let $n \in R$. By Part $1, C\langle\alpha, \beta\rangle$ and $C\left\langle\alpha^{n}, \beta\right\rangle$ are $\mathcal{N} \mathcal{F}$-projectors of $T$, which implies that $C\left\langle\alpha^{n}, \beta\right\rangle=(C\langle\alpha, \beta\rangle)^{\mu}$ for some $\mu \in R$. Since $C\langle\alpha, \beta\rangle$ is a complement in the group $T$ of the normal subgroup $R$, we obtain that $\beta \in C\langle\alpha, \beta\rangle \cap(C\langle\alpha, \beta\rangle)^{\mu} \leq C_{T}(\mu)$. Since $C\langle\alpha, \beta\rangle \cap(C\langle\alpha, \beta\rangle)^{\mu n^{-1}} \leq C_{T}\left(\mu n^{-1}\right)$, it follows that $\alpha \in C_{T}\left(\mu n^{-1}\right)$, and so we have that $n=\left(\mu n^{-1}\right)^{-1} \mu \in C_{R}(\alpha) C_{R}(\beta)$. This means that $R=C_{R}(\alpha) C_{R}(\beta)$. Furthermore, $C_{R}(\alpha) \cap C_{R}(\beta) \leq C \cap R=1$, so we have $R=C_{R}(\alpha) \times C_{R}(\beta)$.

3. Let $n \in N_{1}=\{\mu \in N \mid\langle\mu \alpha, \beta\rangle \in \mathcal{N} \mathcal{F}\}$. We have that $T=N\langle n \alpha, \beta\rangle$ and $\langle n \alpha, \beta\rangle \in \mathcal{N} \mathcal{F}$. Therefore $N\langle n \alpha, \beta\rangle^{\mathcal{F}}=N\langle\alpha, \beta\rangle^{\mathcal{F}}=N O_{p^{\prime}}\left(\langle\alpha, \beta\rangle^{\mathcal{F}}\right)$. Hence we obtain that $N O_{p^{\prime}}\left(\langle n \alpha, \beta\rangle^{\mathcal{F}}\right)=N\langle\alpha, \beta\rangle^{\mathcal{F}}$ and so $C_{N}\left(O_{p^{\prime}}\left(\langle n \alpha, \beta\rangle^{\mathcal{F}}\right)\right)=$ $C$. Arguing as in the proof of Part 1, it follows that $C\langle n \alpha, \beta\rangle$ is an $\mathcal{N} \mathcal{F}$ projector of $T$. By Part 1, $C\langle\alpha, \beta\rangle$ is also an $\mathcal{N} \mathcal{F}$-projector of $T$ and it is a complement of $R$ in $T$. Therefore $C\langle n \alpha, \beta\rangle=(C\langle\alpha, \beta\rangle)^{\rho}$ for some $\rho \in R$. Thus we have $n \alpha=c x^{\rho}$ with $c \in C$ and $x \in\langle\alpha, \beta\rangle$. This means that $n=c\left[\rho, x^{-1}\right] x \alpha^{-1}=\left[\rho, x^{-1}\right] c x \alpha^{-1}$ with $\left[\rho, x^{-1}\right] \in R$ and $c x \alpha^{-1} \in C\langle\alpha, \beta\rangle$. On the other hand, $n \in N=R C$, which implies that $c x \alpha^{-1} \in C$ because $C\langle\alpha, \beta\rangle$ is a complement of $R$ in $T$. Then $x=c_{2} \alpha$ for some $c_{2} \in C$ and so we have $n=c x^{\rho} \alpha^{-1}=c c_{2} \alpha^{\rho} \alpha^{-1} \in C[N,\langle\alpha\rangle]$. This proves that $N_{1} \subseteq C[N,\langle\alpha\rangle]$.

To prove the reverse inclusion, let $n \in C[N,\langle\alpha\rangle]$. By Lemma 7 we may write $n=c \mu^{-1} \alpha \mu \alpha^{-1}$ with $c \in C$ and $\mu \in N$. Therefore $C\langle n \alpha, \beta\rangle=$ $C\left\langle c \alpha^{\mu}, \beta\right\rangle=C\left\langle\alpha^{\mu}, \beta\right\rangle$ and we have $C\langle n \alpha, \beta\rangle^{\mathcal{F}}=C\left\langle\alpha^{\mu}, \beta\right\rangle^{\mathcal{F}}$ because $C$ is a normal subgroup of $T$. We have already seen in the proof of Part 1 that $N\langle\alpha, \beta\rangle^{\mathcal{F}}=N\left\langle\alpha^{\mu}, \beta\right\rangle^{\mathcal{F}}$, so it follows that $C$ centralizes $\left\langle\alpha^{\mu}, \beta\right\rangle^{\mathcal{F}}$. Since $\left\langle\alpha^{\mu}, \beta\right\rangle^{\mathcal{F}} \in \mathcal{N}$, we have $C\langle n \alpha, \beta\rangle^{\mathcal{F}}=C\left\langle\alpha^{\mu}, \beta\right\rangle^{\mathcal{F}} \in \mathcal{N}$. Then $\langle n \alpha, \beta\rangle^{\mathcal{F}} \in \mathcal{N}$ which means that $n \in N_{1}$. We conclude that $N_{1}=C[N,\langle\alpha\rangle]$. Moreover $C[N,\langle\alpha\rangle]=C[C R, \alpha]=C[R, \alpha]$. Similarly $N_{2}=C[N,\langle\beta\rangle]=C[R, \beta]$. 
4. Let $\mu \in N_{1}$. Evidently $N$ is normalized by $\langle\mu \alpha, \beta\rangle$. We have already seen in the proof of Part 3 that $N\langle\mu \alpha, \beta\rangle^{\mathcal{F}}=N O_{p^{\prime}}\left(\langle\alpha, \beta\rangle^{\mathcal{F}}\right)$, so we have that $O_{p}\left(\langle\mu \alpha, \beta\rangle^{\mathcal{F}}\right) \leq N$. Finally, let us see that $\left\langle\mu \alpha, \beta^{n}\right\rangle \in \mathcal{N} \mathcal{F}$ for all $n \in N$. Let $n \in N$. Since $\mu \in N_{1}=C\left[N, \alpha^{-1}\right]$, we may write $\mu=c \rho^{-1} \alpha \rho \alpha^{-1}$ with $c \in C$ and $\rho \in N$. Thus $\mu \alpha=c \alpha^{\rho}$. Arguing as in the proof of Part 3 we have that: $C\left\langle\mu \alpha, \beta^{n}\right\rangle=C\left\langle\alpha^{\rho}, \beta^{n}\right\rangle, N\langle\alpha, \beta\rangle^{\mathcal{F}}=N\left\langle\alpha^{\rho}, \beta^{n}\right\rangle^{\mathcal{F}}, C$ centralizes $\left\langle\alpha^{\rho}, \beta^{n}\right\rangle^{\mathcal{F}}, C\left\langle\mu \alpha, \beta^{n}\right\rangle^{\mathcal{F}}=C\left\langle\alpha^{\rho}, \beta^{n}\right\rangle^{\mathcal{F}} \in \mathcal{N}$ and $\left\langle\mu \alpha, \beta^{n}\right\rangle \in \mathcal{N} \mathcal{F}$.

5. Assume that $L_{1} \leq N_{1}, L_{2} \leq N_{2}$ and $L_{1} L_{2}=N$. We notice now that $N_{1}=C[R, \alpha]$ and $R / C_{R}(\alpha) \cong[R, \alpha]$. By Part $2, R=C_{R}(\alpha) \times C_{R}(\beta)$, so it follows that $\left|N_{1} / C\right|=|[R, \alpha]|=\left|C_{R}(\beta)\right|$. Analogously, $\left|N_{2} / C\right|=$ $|[R, \beta]|=\left|C_{R}(\alpha)\right|$. We have:

$$
\begin{aligned}
|R|=|N / C|=\mid & \left(C L_{1} / C\right)\left(C L_{2} / C\right)|\leq| C L_{1} / C \| C L_{2} / C \mid \leq \\
& \leq\left|N_{1} / C\right|\left|N_{2} / C\right|=|R| .
\end{aligned}
$$

Therefore $\left|C L_{i}\right|=\left|N_{i}\right|$ and so we have that $C L_{i}=N_{i}$ for $i=1,2$.

6. Assume that $\mathcal{A} \subseteq \mathcal{F}$.

First suppose that $N=N_{1}$, we claim that $N=C$. We have that

$$
C \times R=N=N_{1}=C[N,\langle\alpha\rangle]=C[R, \alpha] .
$$

Therefore $R=[R, \alpha]$. Since $R / C_{R}(\alpha) \cong[R, \alpha]=R$ by Lemma 7 , we have that $C_{R}(\alpha)=1$. By Part $2, R=C_{R}(\alpha) C_{R}(\beta)$, so it follows that $R=C_{R}(\beta)$. Since $\mathcal{A} \subseteq \mathcal{F}$, we have $\langle\alpha, \beta\rangle^{\mathcal{F}} \leq\langle\beta\rangle^{\langle\alpha, \beta\rangle} \leq C_{T}(R)$, which implies that $R \leq C$ and so $N=C$ as claimed.

Since $C \leq C[N,\langle\alpha\rangle]=N_{1} \leq N$, we have proved that

$$
N=N_{1} \text { if and only if } N=C \text {. }
$$

Now assume that $\beta$ normalizes $N_{1}$. Let us see that $N=C$. Since $N_{1}=$ $C[N,\langle\alpha\rangle]$, it follows that $N_{1}$ is normalized by $\langle\alpha, \beta\rangle$. In the proof of Part 1 we have seen that $C=C_{N}\left(O_{p^{\prime}}\left(\langle\alpha, \beta\rangle^{\mathcal{F}}\right)\right)$. Then we conclude that $O_{p}\left(\langle\alpha, \beta\rangle^{\mathcal{F}}\right) \leq$ $C \leq N_{1}$. Hence it is clear that $N_{1}, \alpha, \beta$ satisfy Condition $(*)$. Note that $\left\{n \in N_{1} \mid\langle n \alpha, \beta\rangle \in \mathcal{N} \mathcal{F}\right\}=N_{1}$ and so, by the above equivalence we have that $N_{1}=C_{N_{1}}\left(\langle\alpha, \beta\rangle^{\mathcal{F}}\right)=N_{1} \cap C=C$. Therefore $[R,\langle\alpha\rangle] \leq[N,\langle\alpha\rangle] \cap R \leq$ $C \cap R=1$ and so $R=C_{R}(\alpha)$. Arguing as above we have that $R \leq C$ and so $N=C$. Now, Part 6 is clear.

Lemma 9 Let $\mathcal{F}$ be a formation of soluble groups containing all abelian groups. Let $G$ be a soluble group such that $G=A B$ is the $\mathcal{N} \mathcal{F}$-connected product of the subgroups $A$ and $B$. Assume that $\langle a, b\rangle^{\mathcal{F}} \leq F(G \bmod K)$ for all $a \in A, b \in B$ and all non-trivial normal subgroup $K$ of $G$, and assume that there exist $a_{0} \in A$ and $b_{0} \in B$ such that $\left\langle a_{0}, b_{0}\right\rangle^{\mathcal{F}} \not \subset F(G)$.

Then $G$ has a unique minimal normal subgroup $N, N=C_{G}(N)=$ $O_{p}(G)=F(G)$ for a prime $p, N, a, b$ satisfy Condition $(*)$ of Lemma 8 for all $a \in A$ and $b \in B, N \not \subset A$ and $N \not \leq B$. 
Proof. Since $F(G \bmod \Phi(G))=F(G)$, it follows that $\Phi(G)=1$. Let $N$ be a minimal normal subgroup of $G$. Let $a \in A$ and $b \in B$. By the hypothesis we have that $\langle a, b\rangle^{\mathcal{F}} \in \mathcal{N}$. Since $Z:=\langle a, b\rangle^{\mathcal{F}} \leq F(G \bmod N)$, it follows that $Z N \unlhd \unlhd G$. Moreover, $Z N / N \in \mathcal{N}$. Therefore, $[G, k Z] \leq N$ for a suitable $k \geq 1$. If there were another minimal normal subgroup $U$ of $G, N \neq U$, then $[G, l Z] \leq N \cap U=1$, for a suitable $l$. In particular, $Z \unlhd \unlhd G$, which would imply the contradiction $\langle a, b\rangle^{\mathcal{F}} \leq F(G)$ for all $a \in A, b \in B$.

Therefore $G$ is a primitive group. In particular, $N=C_{G}(N)=O_{p}(G)=$ $F(G)$ for a prime $p$, and $F_{2}(G) / N=F(G / N)$ is a $p^{\prime}$-group. Since $\langle a, b\rangle^{\mathcal{F}} \leq$ $F_{2}(G)$, it follows that $O_{p}\left(\langle a, b\rangle^{\mathcal{F}}\right) \leq N$. Hence $N, a, b$ satisfy Condition $(*)$ of Lemma 8.

If either $N \leq A$ or $N \leq B$, then by Lemma 8 (6) it follows that $N=$ $C_{N}\left(\langle a, b\rangle^{\mathcal{F}}\right)$. Consequently $\langle a, b\rangle^{\mathcal{F}} \leq C_{G}(N)=F(G)$ for all $a \in A, b \in B$, which provides a contradiction.

\section{The main result}

Theorem 1 If the soluble group $G=A B$ is the $\mathcal{N}^{2}$-connected product of the subgroups $A$ and $B$, then

$$
G / F(G)=(A F(G) / F(G))(B F(G) / F(G))
$$

is an $\mathcal{N}$-connected product of the two factors.

Proof. We observe first that the statement of the theorem is equivalent to the fact that $\langle a, b\rangle^{\mathcal{N}} \leq F(G)$ for all $a \in A$ and all $b \in B$.

Assume that the result is false and let $G$ be a counterexample with $|G|+|A|+|B|$ minimal. Let $a \in A$ and $b \in B$. By the hypothesis we have that $\langle a, b\rangle \in \mathcal{N}^{2}$ and consequently $\langle a, b\rangle^{\mathcal{N}} \in \mathcal{N}$. The choice of $G$ implies that $\langle a K, b K\rangle^{\mathcal{N}}=\langle a, b\rangle^{\mathcal{N}} K / K \leq F(G / K)$ for all non-trivial normal subgroup $K$ of $G$. By considering $\mathcal{F}=\mathcal{N}$ in Lemma 9 , we obtain that

$G$ has a unique minimal normal subgroup $N$,

$N=C_{G}(N)=O_{p}(G)=F(G)$ for a prime $p$,

$N, a, b$ satisfy Condition $(*)$ of Lemma 8 with $\mathcal{F}=\mathcal{N}$,

$N \not \leq A$ and $N \not \subset B$.

Let us denote $F_{2}=F_{2}(G)=F(G \bmod N)$ and notice that $F_{2} / N$ is a $p^{\prime}$ group. Since $\langle a, b\rangle^{\mathcal{N}} \leq F_{2}$, it follows that

$$
G / F_{2}=\left(A F_{2} / F_{2}\right)\left(B F_{2} / F_{2}\right) \text { is an } \mathcal{N} \text {-connected product. }
$$

We point out also the following fact: 
(3) Whenever $U \leq A, V \leq B$ and $N \leq U V<G$, then $(U N / N)(V N / N)$ is an $\mathcal{N}$-connected product; equivalently, for every pair of primes $l$ and $r$ such that $r \neq l,\left[U_{r}, V_{l}\right] \leq N$ for all $U_{r} \in \operatorname{Syl}_{r}(U)$ and all $V_{l} \in \operatorname{Syl}_{l}(V)$.

To prove this we notice that $F(U V)$ is a $p$-group, since $N=C_{G}(N) \leq$ $F(U V)$ and $N$ is a $p$-group. Now, for any $U_{r}$ and $V_{l}$ as above, we have by the choice of $G$ that $\left[U_{r}, V_{l}\right] \leq F(U V) \cap F_{2} \leq N$.

We split now the proof into two cases:

Case 1: $N A<G$ and $N B<G$.

Case 2: $N A=G$ or $N B=G$.

Case 1: $A N<G$ and $B N<G$.

We claim first:

(1.1) $N=(N \cap A)(N \cap B)$.

Let $X:=X(N)$ the factorizer of $N$ in $A_{p} B_{p}$, for some $A_{p} \in \operatorname{Syl}_{p}(A)$ and $B_{p} \in \operatorname{Syl}_{p}(B)$ such that $A_{p} B_{p} \in \operatorname{Syl}_{p}(G)$. We will show that $X \unlhd \unlhd G$, which implies $N=X$ and proves the claim, as $N \cap A=N \cap A_{p}$ and $N \cap B=N \cap B_{p}$.

We know that

$$
N A_{p}=X A_{p}=\left(X \cap B_{p}\right) A_{p} \text { and } N B_{p}=X B_{p}=\left(X \cap A_{p}\right) B_{p} .
$$

Then

$$
N A=X A=\left(X \cap B_{p}\right) A \text { and } N B=X B=\left(X \cap A_{p}\right) B .
$$

Since $N A<G$, we deduce from (3) that $A N / N$ and $\left(X \cap B_{p}\right) N / N$ are $\mathcal{N}$-connected. In particular, $X=\left(X \cap B_{p}\right) N \unlhd \unlhd A N$. From $N B<G$ we obtain analogously that $X \unlhd \unlhd B N$. Hence $X \unlhd \unlhd(A N)(B N)=G$ (see [13, Theorem 7.7.1]).

Our next aim is to prove that $G$ has the following structure, after interchanging the roles of $A$ and $B$ if necessary:

$$
\begin{aligned}
& A=(N \cap A) A_{q}\langle\alpha\rangle, A_{q} \in \operatorname{Syl}_{q}(A), \alpha \text { an } r \text { - element, } \\
& \alpha \text { normalizes }(N \cap A) A_{q}, \\
& r, q \in \sigma(G), r \neq q \neq p, \\
& B=(N \cap B) B_{q}, B_{q} \in \operatorname{Syl}_{q}(B),\left[B_{q}, \alpha\right] \not \leq N, \\
& A_{q} B_{q} \in \operatorname{Syl}_{q}(G), N A_{q} B_{q} \unlhd G .
\end{aligned}
$$

This is derived in the next two steps by distinguishing the cases when $p|| G: N \mid$ and when $p \nmid|G: N|$. 
(1.2) If $p|| G: N \mid$, then $G$ satisfies (S).

We split the proof of this fact into the following steps:

(1.2.a) $F_{2}=\left(F_{2} \cap A\right)\left(F_{2} \cap B\right)$.

Since $A F_{2}, B F_{2} \unlhd \unlhd G$ from $(2), F\left(A F_{2}\right)=F(G)=F\left(B F_{2}\right)$ and also $F_{2}\left(A F_{2}\right)=F_{2}=F_{2}\left(B F_{2}\right)$.

Assume that $A F_{2}<G$. Since $A F_{2}=A\left(A F_{2} \cap B\right)$, it follows by the choice of $G$ that $A F_{2} / F(G)=(A F(G) / F(G))\left(\left(A F_{2} \cap B\right) F(G) / F(G)\right)$ is an $\mathcal{N}$-connected product. Then by Lemma $2(3)$, we have that

$$
\begin{gathered}
F_{2} / F(G)=F\left(A F_{2} / F(G)\right)= \\
\left(\left(F_{2} / F(G)\right) \cap(A F(G) / F(G))\right)\left(\left(F_{2} / F(G)\right) \cap\left(\left(A F_{2} \cap B\right) F(G) / F(G)\right)\right) \\
=\left(\left(F_{2} \cap A\right) F(G) / F(G)\right)\left(\left(F_{2} \cap B\right) F(G) / F(G)\right),
\end{gathered}
$$

and so

$$
\begin{aligned}
F_{2} & =\left(F_{2} \cap A\right) F(G)\left(F_{2} \cap B\right)= \\
& =\left(F_{2} \cap A\right)(F(G) \cap A)(F(G) \cap B)\left(F_{2} \cap B\right)=\left(F_{2} \cap A\right)\left(F_{2} \cap B\right),
\end{aligned}
$$

as we wanted to prove.

If $B F_{2}<G$, the result follows analogously.

Assume now that $A F_{2}=G=B F_{2}$. Let $A_{p} \in \operatorname{Syl}_{p}(A)$ and $B_{p} \in$ $\operatorname{Syl}_{p}(B)$ such that $A_{p} B_{p} \in \operatorname{Syl}_{p}(G)$. It is clear that $N A_{p}, N B_{p} \in$ $\operatorname{Syl}_{p}(G)$ and so $N A_{p}=N B_{p}=A_{p} B_{p}$. Let us consider

$$
N A=A_{p} N A=B_{p} N A=B_{p}(N \cap B)(N \cap A) A=B_{p} A<G .
$$

By (3) it follows that $A_{p^{\prime}}$ normalizes $B_{p} N=A_{p} N, \forall A_{p^{\prime}} \in \operatorname{Hall}_{p^{\prime}}(A)$. Analogously $B_{p^{\prime}}$ normalizes $A_{p} N=B_{p} N, \forall B_{p^{\prime}} \in \operatorname{Hall}_{p^{\prime}}(B)$. But this implies that $A_{p} N=B_{p} N$ is a normal subgroup of $G$ and so $G / N$ is a $p^{\prime}$-group, a contradiction.

(1.2.b) There exist a prime $q \neq p$, and w.l.o.g. $A_{p} \in \operatorname{Syl}_{p}(A)$ and $B_{q} \in$ $\operatorname{Syl}_{q}(B)$ such that $\left[A_{p}, B_{q}\right] \not \leq N$.

Moreover $G=O_{q} A_{p}$, where $O_{q}:=O_{q}(G \bmod N)$.

If $A_{r} \in \operatorname{Syl}_{r}(A), B_{q} \in \operatorname{Syl}_{q}(B), p \neq q \neq r \neq p$, then $F_{2} A_{r} B_{q}$ is a subgroup of $G$ by (2) and $F_{2} A_{r} B_{q}=A_{r}\left(F_{2} \cap A\right)\left(F_{2} \cap B\right) B_{q}<G$. By (3) we have that $\left[A_{r}, B_{q}\right] \leq N$. Then the first part of the statement follows from Lemma 6 . Moreover $G=F_{2} A_{p} B_{q}$. 
Now Lemma 4 and (1.2.a) imply that

$$
O_{r}:=O_{r}(G \bmod N)=\left(O_{r} \cap A\right)\left(O_{r} \cap B\right) \in \operatorname{Hall}_{\{p, r\}}\left(F_{2}\right),
$$

for any prime $r \neq p$. Hence

$$
\begin{aligned}
& O_{r} \cap A \in \operatorname{Hall}_{\{p, r\}}\left(F_{2} \cap A\right), O_{r} \cap A \unlhd A, \\
& O_{r} \cap B \in \operatorname{Hall}_{\{p, r\}}\left(F_{2} \cap B\right), O_{r} \cap B \unlhd B .
\end{aligned}
$$

In particular, $F_{2} \cap A=\prod_{r \neq p}\left(O_{r} \cap A\right)$ and $F_{2} \cap B=\prod_{r \neq p}\left(O_{r} \cap B\right)$.

We prove next that $G=O_{q} A_{p}$. If $F_{2} A_{p}=G$, then $B_{q} \leq O_{q}$. Hence, if $O_{q} A_{p}=\left(O_{q} \cap B\right)\left(O_{q} \cap A\right) A_{p}<G$, we would deduce from (3) that $\left[A_{p}, B_{q}\right] \leq N$, a contradiction. Thus we can assume that $F_{2} A_{p}<G$.

It follows from the choice of $(G, A, B)$ that $A=A_{p}\left(F_{2} \cap A\right)$ and $B=$ $\left(F_{2} \cap B\right) B_{q}$. In particular,

$$
\begin{aligned}
& \operatorname{Hall}_{\{p, q\}}(A)=\left\{A_{p}^{a}\left(O_{q} \cap A\right) \mid a \in A\right\} \text { and } \\
& \operatorname{Hall}_{\{p, q\}}(B)=\left\{\left(O_{q} \cap B\right) B_{q}^{b} \mid b \in B\right\} .
\end{aligned}
$$

We consider $X_{p} \in \operatorname{Syl}_{p}(A)$ and $Y_{q} \in \operatorname{Syl}_{q}(B)$ such that

$$
T:=X_{p}\left(O_{q} \cap A\right)\left(O_{q} \cap B\right) Y_{q} \in \operatorname{Hall}_{\{p, q\}}(G) .
$$

If $T=G$, then $G=O_{q} X_{p} Y_{q}=O_{q} A_{p} B_{q}$ is a $\{p, q\}$-group. In particular, $F_{2}=O_{q}$. Moreover, $F_{2} B=F_{2} B_{q} \unlhd G$ and $F_{2} B_{q} \in \mathcal{N}^{2}$, that is $B_{q} \leq F_{2}$. This implies that $G=O_{q} A_{p}$ and we are done.

Assume now that $T<G$. By $(3),\left[X_{p}, Y_{q}\right] \leq N$. On the other hand, for any $Q \in \operatorname{Syl}_{q}(B)$, we have that $F_{2} Q=\left(F_{2} \cap A\right)\left(F_{2} \cap B\right) Q<G$ because $p|| G: N \mid$. Then $\left[O_{r} \cap A, Q\right] \leq N$ whenever $r \neq q$. Moreover, for any $P \in \operatorname{Syl}_{p}(A)$, we have $F_{2} P=\left(F_{2} \cap B\right)\left(F_{2} \cap A\right) P<G$ because $F_{2} A_{p}<G$. Then $\left[F_{2} \cap B, P\right] \leq N$.

Now, $A_{p}=X_{p}^{t}$, for some $t=t_{1} t_{2} \in F_{2} \cap A=\left(O_{q} \cap A\right)\left(\prod_{r \neq q}\left(O_{r} \cap A\right)\right)$ with $t_{1} \in O_{q} \cap A, t_{2} \in \prod_{r \neq q}\left(O_{r} \cap A\right)$. Moreover, $B_{q}=Y_{q}^{s}$, for some $s \in F_{2} \cap B$. So it follows that

$$
\begin{aligned}
O_{q} A_{p} B_{q} & =O_{q} X_{p}^{t} Y_{q}^{s}=O_{q} X_{p}^{t_{2}} Y_{q}^{s}=\left(O_{q} X_{p}^{t_{2}} Y_{q}\right)^{s}= \\
& =\left(O_{q} X_{p} Y_{q}\right)^{t_{2} s} \in \operatorname{Hall}_{\{p, q\}}(G) .
\end{aligned}
$$

Consequently, $O_{q} B_{q} A_{p}=B_{q}\left(O_{q} \cap B\right)\left(O_{q} \cap A\right) A_{p}<G$ which implies $\left[A_{p}, B_{q}\right] \leq N$ by (3), a contradiction. 
(1.2.c) $G=O_{q}\langle a\rangle$, for any $a \in A_{p}$ such that $\left[B_{q}, a\right] \not \leq N$. Moreover,

$$
\begin{aligned}
& B=O_{q} \cap B=(B \cap N) B_{q} \text { and } \\
& A=\left(O_{q} \cap A\right)\langle a\rangle=(A \cap N) A_{q}\langle a\rangle, A_{q} \in \operatorname{Syl}_{q}(A) .
\end{aligned}
$$

We can assume $A_{q} B_{q} \in \operatorname{Syl}_{q}(G)$. Also $O_{q}=N A_{q} B_{q} \unlhd G$ and $G$ satisfies $(\mathrm{S})$ with $r=p$.

By (1.2.b) we have that $G=O_{q} A_{p}$ and so $B_{q} \leq O_{q}$. The choice of $(G, A, B)$ implies that $G=O_{q}\langle a\rangle, B=O_{q} \cap B$ and $A=\left(O_{q} \cap A\right)\langle a\rangle$, for any $a$ as in the statement. In particular, $B=(B \cap N) B_{q}$ and $O_{q} \cap A=(N \cap A) A_{q}, A_{q} \in \operatorname{Syl}_{q}(A)$, because $N \in \operatorname{Syl}_{p}\left(O_{q}\right)$.

(1.3) If $p \nmid|G: N|$, then $G$ satisfies (S).

Let us consider $A_{p^{\prime}} \in \operatorname{Hall}_{p^{\prime}}(A)$ and $B_{p^{\prime}} \in \operatorname{Hall}_{p^{\prime}}(B)$ such that $M:=$ $A_{p^{\prime}} B_{p^{\prime}} \in \operatorname{Hall}_{p^{\prime}}(G)$. In this case, $G=N M, M$ is a maximal subgroup of $G$ and $\operatorname{Core}_{G}(M)=1$. We notice the following fact: whenever $X \leq A_{p^{\prime}}$ and $Y \leq B_{p^{\prime}}$, then $X N / N$ and $Y N / N$ are $\mathcal{N}$-connected if and only if $X$ and $Y$ are $\mathcal{N}$-connected. In particular, the choice of $G$ implies that $A_{p^{\prime}}$ and $B_{p^{\prime}}$ are not $\mathcal{N}$-connected.

On the other hand, by the choice of $(G, A, B)$ and taking into account that $N=(N \cap A)(N \cap B)$, the following fact is easily deduced: Whenever $X \leq A_{p^{\prime}}, Y \leq B_{p^{\prime}}, X Y=Y X$ and $|X|+|Y|+|X Y|<$ $\left|A_{p^{\prime}}\right|+\left|B_{p^{\prime}}\right|+|M|$, then $X$ and $Y$ are $\mathcal{N}$-connected.

We set $H=A_{p^{\prime}}$ and $K=B_{p^{\prime}}$. We notice also that, since $M=H K$ is the product of the $\mathcal{N}^{2}$-connected subgroups $H$ and $K$, the choice of $G$ implies that $H F(M) / F(M)$ and $K F(M) / F(M)$ are $\mathcal{N}$-connected.

For every $r \in \sigma(M)$, we consider

$$
\begin{aligned}
\mathcal{C}_{1}=\left\{\left(H_{r^{\prime}}, K_{r}\right) \mid\right. & H_{r^{\prime}} \in \operatorname{Hall}_{r^{\prime}}(H) \text { and there exists } K_{r^{\prime}} \in \operatorname{Hall}_{r^{\prime}}(K) \\
& \text { such that } H_{r^{\prime}} K_{r^{\prime}} \in \operatorname{Hall}_{r^{\prime}}(M) ; \\
& K_{r} \in \operatorname{Syl}_{r}(K) \text { and there exists } H_{r} \in \operatorname{Syl}_{r}(H) \\
& \text { such that } \left.H_{r} K_{r} \in \operatorname{Syl}_{r}(M)\right\}
\end{aligned}
$$

and

$$
\begin{aligned}
\mathcal{C}_{2}=\left\{\left(K_{r^{\prime}}, H_{r}\right) \mid\right. & K_{r^{\prime}} \in \operatorname{Hall}_{r^{\prime}}(K) \text { and there exists } H_{r^{\prime}} \in \operatorname{Hall}_{r^{\prime}}(H) \\
& \text { such that } H_{r^{\prime}} K_{r^{\prime}} \in \operatorname{Hall}_{r^{\prime}}(M) ; \\
& H_{r} \in \operatorname{Syl}_{r}(H) \text { and there exists } K_{r} \in \operatorname{Syl}_{r}(K) \\
& \text { such that } \left.H_{r} K_{r} \in \operatorname{Syl}_{r}(M)\right\} .
\end{aligned}
$$

It is known that $\mathcal{C}_{1} \neq \emptyset$ and $\mathcal{C}_{2} \neq \emptyset$. The following steps lead now to the desired structure of $G$. 
(1.3.a) There exist $r \in \sigma(M)$ and w.l.o.g. $\left(H_{r^{\prime}}, K_{r}\right) \in \mathcal{C}_{1}$ such that $H_{r^{\prime}} K_{r} F(M)=M$.

Moreover,

$$
\begin{aligned}
& W:=F\left(H_{r^{\prime}} K_{r^{\prime}}\right)=\left(W \cap H_{r^{\prime}}\right)\left(W \cap K_{r^{\prime}}\right) \text { and } \\
& F(M)=O_{r^{\prime}}(F(M)) O_{r}(F(M)) \leq W O_{r}(F(M))=W F(M) .
\end{aligned}
$$

Assume that for every $r \in \sigma(M)$, we have $H_{r^{\prime}} K_{r} F(M)<M$ and $K_{r^{\prime}} H_{r} F(M)<M$ for all $\left(H_{r^{\prime}}, K_{r}\right) \in \mathcal{C}_{1}$ and $\left(K_{r^{\prime}}, H_{r}\right) \in \mathcal{C}_{2}$. Let $r \in \sigma(M)$. We claim that $H_{r^{\prime}} K_{r} F(M)<M,\left(H_{r^{\prime}}, K_{r}\right) \in \mathcal{C}_{1}$, implies that $\left[H_{r^{\prime}}, K_{r}\right]=1$. We consider $X_{r}:=O_{r}(F(M)) K_{r} \leq H_{r} K_{r}$ and $Y_{r^{\prime}}:=O_{r^{\prime}}(F(M)) H_{r^{\prime}} \leq H_{r^{\prime}} K_{r^{\prime}}$. Clearly $X_{r}=\left(X_{r} \cap H_{r}\right) K_{r}$ and $Y_{r^{\prime}}=H_{r^{\prime}}\left(Y_{r^{\prime}} \cap K_{r^{\prime}}\right)$. Then

$$
\begin{aligned}
R: & =H_{r^{\prime}} K_{r} F(M)=H_{r^{\prime}} O_{r^{\prime}}(F(M)) O_{r}(F(M)) K_{r}= \\
& =H_{r^{\prime}}\left(Y_{r^{\prime}} \cap K_{r^{\prime}}\right)\left(X_{r} \cap H_{r}\right) K_{r}
\end{aligned}
$$

contains $S:=\left\langle H_{r^{\prime}}, X_{r} \cap H_{r}\right\rangle\left\langle Y_{r^{\prime}} \cap K_{r^{\prime}}, K_{r}\right\rangle$.

We set $S_{1}=\left\langle H_{r^{\prime}}, X_{r} \cap H_{r}\right\rangle$ and $S_{2}=\left\langle Y_{r^{\prime}} \cap K_{r^{\prime}}, K_{r}\right\rangle$. We notice that

$$
\left|S_{1} \cap S_{2}\right| \leq|H \cap K|=|H \cap K|_{r}|H \cap K|_{r^{\prime}}=\left|H_{r} \cap K_{r}\right|\left|H_{r^{\prime}} \cap K_{r^{\prime}}\right|
$$

because $H_{r^{\prime}} K_{r^{\prime}} \in \operatorname{Hall}_{r^{\prime}}(M), H_{r} K_{r} \in \operatorname{Syl}_{r}(M)$ and $M=H K$. Consequently,

$$
\begin{aligned}
|S| & =\frac{\left|S_{1}\right|\left|S_{2}\right|}{\left|S_{1} \cap S_{2}\right|} \geq \frac{\left|H_{r^{\prime}}\right|\left|X_{r} \cap H_{r}\right|\left|Y_{r^{\prime}} \cap K_{r^{\prime}}\right|\left|K_{r}\right|}{\left|S_{1} \cap S_{2}\right|} \geq \\
& \geq \frac{\left|H_{r^{\prime}}\right|\left|Y_{r^{\prime}} \cap K_{r^{\prime}}\right|\left|X_{r} \cap H_{r}\right|\left|K_{r}\right|}{\left|H_{r} \cap K_{r}\right|\left|H_{r^{\prime}} \cap K_{r^{\prime}}\right|}=|R| .
\end{aligned}
$$

Since $S \subseteq R$, we deduce that $S=R<M$. Moreover $S=S_{1} S_{2}$, $S_{1} \leq H$ and $S_{2} \leq K$. By the choice of $(G, A, B)$ we have that $S_{1}$ and $S_{2}$ are $\mathcal{N}$-connected. In particular $\left[H_{r^{\prime}}, K_{r}\right]=1$, as claimed.

Let $\left(H_{r^{\prime}}, K_{r}\right) \in \mathcal{C}_{1}$. We have now that $H K_{r}=H_{r^{\prime}} H_{r} K_{r}=H_{r^{\prime}} K_{r} H_{r}=$ $K_{r} H \leq M$. We prove next that $H$ and $K_{r}$ are $\mathcal{N}$-connected. If $|H|+\left|K_{r}\right|+\left|H K_{r}\right|<|H|+|K|+|M|$, then the result is true by the choice of $(G, A, B)$. So we may assume that $H K_{r}=M$ and $K=K_{r}$. Let $Q \in \operatorname{Hall}_{r^{\prime}}(H)$. We notice that in this case $\left(Q, K_{r}\right) \in \mathcal{C}_{1}$, which implies $\left[Q, K_{r}\right]=1$ by the initial assumption. But this means that $H$ and $K_{r}$ are $\mathcal{N}$-connected by Lemma 6 .

By what we have shown, $\left[H^{\mathcal{N}}, K_{r}\right]=1$ for all $r \in \sigma(M)$. Consequently, for all $r \in \sigma(M)$ and every pair $\left(H_{r^{\prime}}, K_{r}\right) \in \mathcal{C}_{1}$, we have $\left[H^{\mathcal{N}}, K\right]=1$ 
and $\left[H_{r^{\prime}}, K_{r}\right]=1$. In an analogous way we deduce $\left[K^{\mathcal{N}}, H\right]=1$ and $\left[K_{r^{\prime}}, H_{r}\right]=1$ for all $r \in \sigma(M)$ and every $\left(K_{r^{\prime}}, H_{r}\right) \in \mathcal{C}_{2}$. Lemma 6 implies now that $H$ and $K$ are $\mathcal{N}$-connected, a contradiction. This proves the first part of (1.3.a).

For the second part, since $r \in \sigma(M), H_{r^{\prime}} K_{r^{\prime}}<M$ and so $H_{r^{\prime}}$ and $K_{r^{\prime}}$ are $\mathcal{N}$-connected. Consequently, $W=F\left(H_{r^{\prime}} K_{r^{\prime}}\right)=\left(W \cap H_{r^{\prime}}\right)(W \cap$ $\left.K_{r^{\prime}}\right)$ by Lemma $2(3)$. The rest is clear.

(1.3.b) $W F(M) \leq H_{r^{\prime}} K_{r^{\prime}} F(M)=H_{r^{\prime}} F(M)$ and $W F(M) K_{r} \leq M$.

By the choice of $G$ we have that $H_{r^{\prime}} F(M) / F(M)$ and $K_{r} F(M) / F(M)$ are $\mathcal{N}$-connected, which implies that $H_{r^{\prime}} F(M) \unlhd M$ and $H_{r^{\prime}} K_{r^{\prime}} \leq$ $H_{r^{\prime}} F(M)$ by (1.3.a). In particular we have $W F(M) \leq H_{r^{\prime}} K_{r^{\prime}} F(M)=$ $H_{r^{\prime}} F(M)$ and $W F(M)=\left(W F(M) \cap H_{r^{\prime}}\right) F(M)$. Consequently, it follows that $W F(M) K_{r}=\left(W F(M) \cap H_{r^{\prime}}\right) K_{r} F(M) \leq M$ because $H_{r^{\prime}} F(M) / F(M)$ and $K_{r} F(M) / F(M)$ are $\mathcal{N}$-connected.

(1.3.c) If $W F(M) K_{r}<M$, then $F(M)=(F(M) \cap H)(F(M) \cap K)$.

We notice that $H_{r^{\prime}}$ normalizes $F(M) K_{r}$ and also $W$. Since $M=$ $H_{r^{\prime}} K_{r} F(M)$ by (1.3.a), we deduce that $W F(M) K_{r} \unlhd M$, and so $F(M)=F\left(W F(M) K_{r}\right)$. Moreover, $O_{r}(F(M)) K_{r}=H_{r} K_{r}$ because $O_{r}(F(M)) K_{r} \in \operatorname{Syl}_{r}(M)$ and $O_{r}(F(M)) K_{r} \leq H_{r} K_{r}$. By (1.3.a) it follows now that

$$
\begin{aligned}
& W F(M) K_{r}=\left(W \cap H_{r^{\prime}}\right)\left(W \cap K_{r^{\prime}}\right) O_{r}(F(M)) K_{r}= \\
& =\left(W \cap H_{r^{\prime}}\right)\left(W \cap K_{r^{\prime}}\right) H_{r} K_{r} \supseteq\left\langle W \cap H_{r^{\prime}}, H_{r}\right\rangle\left\langle W \cap K_{r^{\prime}}, K_{r}\right\rangle .
\end{aligned}
$$

Let $T_{1}:=\left\langle W \cap H_{r^{\prime}}, H_{r}\right\rangle$ and $T_{2}:=\left\langle W \cap K_{r^{\prime}}, K_{r}\right\rangle$. We notice again that $\left|T_{1} \cap T_{2}\right| \leq|H \cap K|=\left|H_{r^{\prime}} \cap K_{r^{\prime}}\right|\left|H_{r} \cap K_{r}\right|$. Moreover $H_{r^{\prime}} \cap K_{r^{\prime}} \leq W$ because $H_{r^{\prime}} K_{r^{\prime}}$ is an $\mathcal{N}$-connected product. In particular, $W \cap H_{r^{\prime}} \cap$ $K_{r^{\prime}}=H_{r^{\prime}} \cap K_{r^{\prime}}$. Consequently,

$$
\begin{aligned}
&\left|T_{1} T_{2}\right|=\frac{\left|T_{1}\right|\left|T_{2}\right|}{\left|T_{1} \cap T_{2}\right|} \geq \frac{\left|W \cap H_{r^{\prime}}\right|\left|H_{r}\right|\left|W \cap K_{r^{\prime}}\right|\left|K_{r}\right|}{\left|H_{r^{\prime}} \cap K_{r^{\prime}}\right|\left|H_{r} \cap K_{r}\right|}= \\
&=\frac{\left|W \cap H_{r^{\prime}}\right|\left|W \cap K_{r^{\prime}}\right|}{\left|W \cap H_{r}\right|\left|K_{r}\right|}=\left|W F(M) K_{r^{\prime}} \cap K_{r^{\prime}}\right| \\
&\left|H_{r} \cap K_{r}\right|
\end{aligned}
$$

Since $T_{1} T_{2} \subseteq W F(M) K_{r}$ we have that $W F(M) K_{r}=T_{1} T_{2}$. But $T_{1} \leq$ $H, T_{2} \leq K$ and $T_{1} T_{2}<M$. Then the choice of $(G, A, B)$ implies that $T_{1}$ and $T_{2}$ are $\mathcal{N}$-connected. Consequently,

$$
\begin{aligned}
F(M) & =F\left(W F(M) K_{r}\right)=\left(F(M) \cap T_{1}\right)\left(F(M) \cap T_{2}\right) \subseteq \\
& \subseteq(F(M) \cap H)(F(M) \cap K) \subseteq F(M) .
\end{aligned}
$$

This means that $F(M)=(F(M) \cap H)(F(M) \cap K)$ and we are done. 
(1.3.d) If $W F(M) K_{r}=M$, then $F(M)=(F(M) \cap H)(F(M) \cap K)$.

In this case we have that $M / F(M) \in \mathcal{N}$. This is because $\left[K_{r}, H_{r^{\prime}}\right] \leq$ $F(M)$, which implies $\left[K_{r}, W\right] \leq F(M)$, since $W \leq H_{r^{\prime}} F(M)$ by (1.3.b).

Let $X:=X(F(M))$ be the factorizer of $F(M)$ in $M=H K$. Then $F(M) \leq X=(X \cap H)(X \cap K)$. Since $M / F(M) \in \mathcal{N}$, it follows that $X \unlhd \unlhd M$ and $F(M)=F(X)$.

If $X<M$, then $X \cap H$ and $X \cap K$ are $\mathcal{N}$-connected by the choice of $(G, A, B)$. Therefore

$F(X)=(F(X) \cap X \cap H)(F(X) \cap X \cap K)=(F(X) \cap H)(F(X) \cap K)$ and $F(M)=F(X)$ yields the assertion.

Assume that $X=M=F(M) H=F(M) K$. Let $l \in \sigma(M)$ and let $H_{l} \in \operatorname{Syl}_{l}(H)$ and $K_{l} \in \operatorname{Syl}_{l}(K)$ such that $H_{l} K_{l} \in \operatorname{Syl}_{l}(M)$.

We notice that $O_{l}(M) H_{l}=O_{l}(M) K_{l}=H_{l} K_{l}$ because $H_{l} K_{l}, O_{l}(M) H_{l}$, $O_{l}(M) K_{l} \in \operatorname{Syl}_{l}(M)$ and both $O_{l}(M) H_{l}$ and $O_{l}(M) K_{l}$ are contained in $H_{l} K_{l}$.

On the other hand, since $M / F(M) \in \mathcal{N}$, we deduce that $H^{\mathcal{N}} \leq M^{\mathcal{N}} \cap$ $H \leq F(M) \cap H$ and, consequently,

$$
H=\prod_{l \in \sigma(H)}(F(M) \cap H) H_{l}
$$

as $(F(M) \cap H) H_{l} \unlhd H$ for all $l \in \sigma(H)$. Moreover,

$$
F(M) \cap H=O_{l^{\prime}}(F(M) \cap H) \times O_{l}(F(M) \cap H),
$$

$O_{l^{\prime}}(F(M) \cap H) \unlhd H$ and $O_{l}(F(M) \cap H) \leq H_{l}$.

In particular, $H_{l}$ normalizes $O_{l^{\prime}}(F(M) \cap H)$. Since $O_{l}(M)$ centralizes $O_{l^{\prime}}(F(M) \cap H)$, we can deduce that $H_{l} K_{l}=O_{l}(M) H_{l}$ normalizes $O_{l^{\prime}}(F(M) \cap H)$. In particular, $O_{l^{\prime}}(F(M) \cap H) K_{l} \leq M$.

If $M=O_{l^{\prime}}(F(M) \cap H) K_{l}$, then

$$
\begin{aligned}
F(M) & =O_{l^{\prime}}(F(M) \cap H)\left(F(M) \cap K_{l}\right) \subseteq \\
& \subseteq(F(M) \cap H)(F(M) \cap K) \subseteq F(M),
\end{aligned}
$$

that is, $F(M)=(F(M) \cap H)(F(M) \cap K)$ and (1.3.d) is proved.

If $O_{l^{\prime}}(F(M) \cap H) K_{l}<M$, then $O_{l^{\prime}}(F(M) \cap H)$ and $K_{l}$ are $\mathcal{N}$ connected. In particular, $\left[O_{l^{\prime}}(F(M) \cap H), K_{l}\right]=1$. Since $O_{l}(M)$ centralizes $O_{l^{\prime}}(F(M) \cap H)$ and $H_{l} K_{l}=O_{l}(M) K_{l}$, we deduce now that $\left[O_{l^{\prime}}(F(M) \cap H), H_{l}\right]=1$. But this means that

$$
(F(M) \cap H) H_{l}=O_{l^{\prime}}(F(M) \cap H) H_{l} \in \mathcal{N} .
$$


Consequently,

$$
H=\prod_{l \in \sigma(H)}(F(M) \cap H) H_{l} \in N_{0} \mathcal{N}=\mathcal{N} .
$$

In an analogous way we can assume that $K \in \mathcal{N}$. But this implies that $F(M)=(F(M) \cap H)(F(M) \cap K)$ by [1, Lemma 2.5.7] and the step is proved.

(1.3.e) $F(M)=(F(M) \cap H)(F(M) \cap K)$. In particular,

$$
O_{s}(M)=\left(O_{s}(M) \cap H\right)\left(O_{s}(M) \cap K\right) \text { for all } s \in \sigma(F(M)) .
$$

This follows from (1.3.c), (1.3.d) and Lemma 4.

We take now $1 \neq x \in H, x$ an $l$-element, $1 \neq y \in K, y$ a $q$-element, $l \in \sigma(H), q \in \sigma(K), l \neq q$, such that $[x, y] \neq 1$, whose existence is assured by Lemma 6 .

(1.3.f) $M=F(M)\langle x\rangle\langle y\rangle, \sigma(M)=\{l, q\}$ and w.l.o.g. one of the following cases holds:

I. $O_{q}(M)\langle x\rangle=M$

II. $F(M)\langle x\rangle<M$ and $F(M)\langle y\rangle<M$.

Since $H F(M) / F(M)$ and $K F(M) / F(M)$ are $\mathcal{N}$-connected, it is clear that $[x, y] \in F(M)$. Then

$F(M)\langle x, y\rangle=F(M)\langle x\rangle\langle y\rangle=\langle x\rangle(F(M) \cap H)(F(M) \cap K)\langle y\rangle$,

where $\langle x\rangle(F(M) \cap H) \leq H$ and $(F(M) \cap K)\langle y\rangle \leq K$. By the choice of $G$ it follows that $F(M)\langle x\rangle\langle y\rangle=M$.

Assume that the case II does not hold and w.l.o.g. $F(M)\langle x\rangle=M$. Then $y \in O_{q}(M) \cap K$. Again the choice of $G$ implies that $O_{q}(M)\langle x\rangle=$ $\left(O_{q}(M) \cap K\right)\left(O_{q}(M) \cap H\right)\langle x\rangle=M$ and I holds.

We prove next that $\sigma(M)=\{l, q\}$. This is clear in the case I. Assume that II holds. Let

$$
K_{1}:=\langle y\rangle\left(O_{q}(M) \cap K\right)\left(\times_{s \neq q, l}\left(O_{s}(M) \cap K\right)\right)\left(O_{l}(M) \cap K\right)
$$

and

$$
H_{1}:=\left(O_{q}(M) \cap H\right)\left(\times_{s \neq q, l}\left(O_{s}(M) \cap H\right)\right)\left(O_{l}(M) \cap H\right)\langle x\rangle .
$$

Since $M=F(M)\langle x\rangle\langle y\rangle$, it is clear that $M=H_{1} K_{1}$ and so $K=K_{1}$ and $H=H_{1}$ by the choice of $(G, A, B)$. Let us consider $F(M)\langle x\rangle=$ 
$(F(M) \cap K)(F(M) \cap H) Q<M$, for any $Q \in \operatorname{Hall}_{\{q, l\}}(H)$. By the choice of $G$ we have in particular that $\left[\times_{s \neq q, l}\left(O_{s}(M) \cap K\right), Q\right]=1$. From $F(M)\langle y\rangle<M$, we deduce that $\left[\times_{s \neq q, l}\left(O_{s}(M) \cap H\right), P\right]=1$ for any $P \in \operatorname{Hall}_{\{q, l\}}(K)$. We consider now $X \in \operatorname{Hall}_{\{q, l\}}(K)$ and $Y \in$ $\operatorname{Hall}_{\{q, l\}}(H)$ such that $X Y=Y X \in \operatorname{Hall}_{\{q, l\}}(M)$. Then we have that

$$
X_{1}:=X^{u}=\langle y\rangle\left(O_{q}(M) \cap K\right)\left(O_{l}(M) \cap K\right) \leq K,
$$

for some $u \in \times_{s \neq q, l}\left(O_{s}(M) \cap K\right)$, and

$$
Y_{1}:=Y^{v}=\left(O_{q}(M) \cap H\right)\left(O_{l}(M) \cap H\right)\langle x\rangle \leq H,
$$

for some $v \in \times_{s \neq q, l}\left(O_{s}(M) \cap H\right)$. Moreover

$$
X_{1} Y_{1}=X^{u} Y^{v}=X^{u v} Y^{u v}=(X Y)^{u v} \leq M .
$$

If $|\sigma(M)| \geq 3$, then $X_{1} Y_{1}<M$ and we would obtain the contradiction $[x, y]=1$. Therefore $\sigma(M)=\{l, q\}$ and we are done.

(1.3.g) (1.3.f)I holds and $G$ satisfies (S) with $r=l$.

Assume that $M=F(M)\langle x\rangle\langle y\rangle, \sigma(M)=\{l, q\}, F(M)\langle x\rangle<M$ and $F(M)\langle y\rangle<M$. By the choice of $(G, A, B)$ we have that $H=\left(O_{q}(M) \cap\right.$ $H)\left(O_{l}(M) \cap H\right)\langle x\rangle$ and $K=\langle y\rangle\left(O_{q}(M) \cap K\right)\left(O_{l}(M) \cap K\right)$.

We claim that $H \unlhd \unlhd M$ and $K \unlhd \unlhd M$. Since $H F(M) / F(M)$ and $K F(M) / F(M)$ are $\mathcal{N}$-connected, we have $H F(M) \unlhd \unlhd M$. Moreover $F(M) H=(F(M) \cap K) H=F(M)\langle x\rangle<M$, which implies that $(F(M) \cap K)$ and $H$ are $\mathcal{N}$-connected by the choice of $G$. In particular, $H \unlhd \unlhd F(M) H$ and so $H \unlhd \unlhd M$. Analogously, $K \unlhd \unlhd M$. Consequently $M^{\mathcal{N}}=(H K)^{\mathcal{N}}=H^{\mathcal{N}} K^{\mathcal{N}}$ by Lemma 1 (3). But $H^{\mathcal{N}} \leq O_{q}(M) \cap H$ and $K^{\mathcal{N}} \leq O_{l}(M) \cap K$, which implies $H^{\mathcal{N}}=O_{q}\left(M^{\mathcal{N}}\right)$ and $K^{\mathcal{N}}=O_{l}\left(M^{\mathcal{N}}\right)$ since $M / F(M) \in \mathcal{N}$. In particular, $O_{q}\left(\langle x, y\rangle^{\mathcal{N}}\right) \leq O_{q}\left(M^{\mathcal{N}}\right) \leq H$ and $O_{l}\left(\langle x, y\rangle^{\mathcal{N}}\right) \leq O_{l}\left(M^{\mathcal{N}}\right) \leq K$. Then

$$
\langle x, y\rangle=\langle x\rangle\langle x, y\rangle^{\mathcal{N}}\langle y\rangle=\langle x\rangle O_{q}\left(\langle x, y\rangle^{\mathcal{N}}\right) O_{l}\left(\langle x, y\rangle^{\mathcal{N}}\right)\langle y\rangle,
$$

where $\langle x\rangle O_{q}\left(\langle x, y\rangle^{\mathcal{N}}\right) \leq H$ and $O_{l}\left(\langle x, y\rangle^{\mathcal{N}}\right)\langle y\rangle \leq K$. It follows that $M=\langle x, y\rangle, H=\langle x\rangle O_{q}\left(M^{\mathcal{N}}\right)$ and $K=O_{l}\left(M^{\mathcal{N}}\right)\langle y\rangle$, by the choice of $(G, A, B)$.

We notice now that $N, x, y$ satisfy Condition $(*)$ of Lemma 8. In particular $C_{N}\left(\langle x, y\rangle^{\mathcal{N}}\right)\langle x, y\rangle$ is an $\mathcal{N}^{2}$-projector of $G$, which implies $C_{N}\left(\langle x, y\rangle^{\mathcal{N}}\right)=1$. Moreover $N=(N \cap A)(N \cap B)$. By Lemma 8 (5) we deduce that $N \cap A=[N,\langle x\rangle]$ and $N \cap B=[N,\langle y\rangle]$. In particular it 
follows that $N \cap B=\left[N,\langle y\rangle^{K}\right]$. But $K^{\mathcal{N}} \leq\langle y\rangle^{K}$ and $K^{\mathcal{N}}=O_{l}\left(M^{\mathcal{N}}\right)$ is a normal subgroup of $M$. Consequently, $\left[N, K^{\mathcal{N}}\right] \leq N \cap B<N$, because $N \not \subset B$, and $\left[N, K^{\mathcal{N}}\right] \unlhd G=N M$. This implies that $\left[N, K^{\mathcal{N}}\right]=1$ and so $K^{\mathcal{N}} \leq C_{G}(N)=N$, that is, $K^{\mathcal{N}}=1$. Analogously we deduce that $H^{\mathcal{N}}=1$. But $M^{\mathcal{N}}=H^{\mathcal{N}} K^{\mathcal{N}}=1$, a contradiction. This proves that (1.3.f)I holds and the choice of $(G, A, B)$ provides the desired structure for $G$.

Assuming the structure (S) for the group $G$, let $\beta \in B_{q}$ such that $[\alpha, \beta] \notin N$, $C:=C_{N}\left(\langle\alpha, \beta\rangle^{\mathcal{N}}\right)$ and $L:=N A_{q} \Phi\left(A_{q} B_{q}\right) \unlhd G$. We denote by bars the images in the factor group $\bar{G}=G / N$. The final contradiction for case 1 is derived next:

\section{(1.4) $L \cap B_{q}=1$.}

We claim first that $\left[L \cap B_{q}, \alpha\right] \leq N$. Since $A \leq L\langle\alpha\rangle \leq A B=G$, it is clear that $L\langle\alpha\rangle=A(L\langle\alpha\rangle \cap B)$. If $L\langle\alpha\rangle=G$, we obtain the contradiction $G=N A_{q}\langle\alpha\rangle=N A$, because $\Phi\left(\overline{A_{q} B_{q}}\right) \leq \Phi(\bar{G})$. Consequently the claim follows by (3).

Assume that $L \cap B_{q} \neq 1$. Since $L \cap B_{q} \unlhd B_{q}$, there exists $1 \neq z \in$ $L \cap B_{q} \cap Z\left(B_{q}\right)$. We notice now that $N, \alpha, \beta$ and also $N, \alpha, z \beta$ satisfy Condition $(*)$ of Lemma 8. Moreover,

$$
C_{N}\left(\langle\alpha, z \beta\rangle^{\mathcal{N}}\right)=C_{N}(N[\langle\alpha\rangle,\langle z \beta\rangle])=C_{N}(N[\langle\alpha\rangle,\langle\beta\rangle])=C,
$$

because $[\alpha, z] \in N$ by the previous claim. By Lemma 8 , we deduce in particular that

$$
C(N \cap B)=C[N,\langle\beta\rangle]=C[N,\langle z \beta\rangle]
$$

as $N=(N \cap A)(N \cap B)$ by (1.1). Hence $[z, N] \leq(N \cap B) C$.

If $(N \cap B) C<N$, then $C_{N}(z) \neq 1$ by coprime action since $z$ is a $q$-element, $q \neq p$. But $N\left\langle B_{q}, \alpha\right\rangle=G$ by (3), which implies that $z \in Z(G \bmod N)$. Hence $C_{N}(z)=N$, but this means $z \in C_{G}(N)=N$, a contradiction.

Therefore $C[N,\langle\beta\rangle]=N$. By Lemma 8 (3) and (6) it follows that $N=C$, which implies $[\alpha, \beta] \in N$, a contradiction. This proves that $L \cap B_{q}=1$.

(1.5) $A_{q}=1$.

We have $N A_{q} \leq L \leq A_{q} B_{q} N$. Consequently, by (1.4), $L=N A_{q}\left(B_{q} \cap\right.$ $L)=N A_{q}$, whence $N A_{q} \unlhd G$ and $A_{q} \unlhd A_{q} B_{q}$. Assume that $A_{q} \neq 1$. 
Then $1 \neq S:=A_{q} \cap Z\left(A_{q} B_{q}\right)$. Let $s \in S$. By coprime action we have that $\bar{S}=C_{\bar{S}}(\alpha)[\bar{S},\langle\alpha\rangle]$. Then $\bar{s}=\overline{s_{1}} \overline{s_{2}}, \overline{s_{1}} \in C_{\bar{S}}(\alpha), \overline{s_{2}} \in$ $[\bar{S},\langle\alpha\rangle], s_{1}, s_{2} \in S$. Moreover, since $\bar{S}$ is abelian, $[\bar{S},\langle\alpha\rangle]=\left[\bar{S}, \alpha^{-1}\right]=$ $\left\{\bar{\sigma}^{-1} \bar{\sigma}^{\alpha^{-1}} \mid \bar{\sigma} \in \bar{S}\right\}$. In particular, $\bar{s}_{2}=\bar{\sigma}^{-1} \bar{\sigma}^{\alpha^{-1}}$, for some $\bar{\sigma} \in \bar{S}$. Then $\bar{s}_{2} \bar{\alpha}=\bar{\alpha} \bar{\sigma}$, with $\bar{\sigma} \in \bar{S}$, is an $r$-element centralized by $\bar{s}_{1} \in Z\left(\overline{A_{q} B_{q}}\right)$. Hence $\langle\bar{\beta}, \bar{s} \bar{\alpha}\rangle=\left\langle\bar{\beta}, \bar{s}_{1}, \bar{s}_{2} \bar{\alpha}\right\rangle=\left\langle\bar{s}_{1}\right\rangle\left\langle\bar{\beta}, \bar{s}_{2} \bar{\alpha}\right\rangle$, which is a central product. Consequently,

$$
\begin{aligned}
\langle\beta, s \alpha\rangle^{\mathcal{N}} N / N & =\langle\bar{\beta}, \bar{s} \bar{\alpha}\rangle^{\mathcal{N}}=\left\langle\bar{\beta}, \bar{s}_{2} \bar{\alpha}\right\rangle^{\mathcal{N}}=\left\langle\bar{\beta}, \bar{\alpha}^{\bar{\sigma}}\right\rangle^{\mathcal{N}}= \\
& =\left(\langle\bar{\beta}, \bar{\alpha}\rangle^{\mathcal{N}}\right)^{\bar{\sigma}}=\langle\bar{\beta}, \bar{\alpha}\rangle^{\mathcal{N}}=\langle\beta, \alpha\rangle^{\mathcal{N}} N / N .
\end{aligned}
$$

Then it is clear that $C=C_{N}\left(\langle\beta, s \alpha\rangle^{\mathcal{N}}\right)$. By Lemma 8 applied to $N, \alpha, \beta$ and $N, s \alpha, \beta$ we have in particular that

$$
C(N \cap A)=C[N,\langle\alpha\rangle]=C[N,\langle s \alpha\rangle] .
$$

Therefore we have that $[N, S] \leq(N \cap A) C$. Since $S$ is a $q$-group and $N S \unlhd G$ we can argue as in (1.4) to deduce that $C[N,\langle\alpha\rangle]=N$. But again Lemma 8 (3) and (6) implies that $N=C$, which yields the contradiction $[\alpha, \beta] \in N$.

(1.6) Final contradiction for case 1.

By the structure (S) of $G,(1.4)$ and (1.5) we have that $N B_{q} \unlhd G=$ $N B_{q}\langle\alpha\rangle, \Phi\left(B_{q}\right) \leq L \cap B_{q}=1$ and $\alpha$ is an $r$-element, $r \neq q$. Hence $\bar{B}_{q}$ is a completely reducible $\langle\alpha\rangle$-module over $\operatorname{GF}(q)$. If $\bar{V}$ is a proper $\langle\alpha\rangle$ submodule of $\bar{B}_{q}, V<B_{q}$, we can consider $N V\langle\alpha\rangle=V(N \cap B)(N \cap$ $A)\langle\alpha\rangle<G$ and deduce that $[V, \alpha] \leq N$ by (3). But this implies that $\bar{B}_{q}$ is an irreducible $\operatorname{GF}(q)\langle\alpha\rangle$-module, because otherwise $\left[B_{q}, \alpha\right] \leq N$, a contradiction. It follows in particular that

$$
G=N\langle\beta, \alpha\rangle=N\left\langle\beta^{\alpha}, \alpha\right\rangle=N\left\langle\beta_{1}, \alpha\right\rangle,
$$

for $\beta_{1} \in B_{q}$ such that $\beta^{\alpha}=n \beta_{1}$, for some $n \in N$. We notice that $C=C_{N}\left(\left\langle\beta_{1}, \alpha\right\rangle^{\mathcal{N}}\right)=1$. By Lemma 8 we obtain that

$$
[N, \beta]=N \cap B=\left[N, \beta_{1}\right]=\left[N, n \beta_{1}\right]=\left[N, \beta^{\alpha}\right]=[N, \beta]^{\alpha},
$$

which implies by Lemma 8 (6) that $N=C=1$, the final contradiction.

Case 2: $A N=G$ or $B N=G$.

We may assume that $A N=G$. Then $A$ is a maximal subgroup of $G$ and $A \cap N=1$.

Let $A_{p} \in \operatorname{Syl}_{p}(A)$ and $B_{p} \in \operatorname{Syl}_{p}(B)$ such that $A_{p} B_{p} \in \operatorname{Syl}_{p}(G)$. We consider again $X=X(N)$ the factorizer of $N$ in $A_{p} B_{p}$. Then we have $\left(X \cap A_{p}\right)\left(X \cap B_{p}\right)=X=N A_{p} \cap N B_{p}$. Moreover, recall that $N \not \leq A$ and $N \not \leq B$ by (1). 
We will derive a contradiction in this case by means of the following steps:

(2.1) $B=X \cap B_{p}$ is a $p$-group, $N B=X=\left(X \cap A_{p}\right) B$ and $X \cap A_{p} \neq 1$.

Suppose that $X \cap B_{p}<B$. Since $G=A N=A X=A\left(X \cap B_{p}\right)$, by the choice of $(G, A, B)$ we obtain that $G / N$ is the product of the $\mathcal{N}$-connected subgroups $A N / N$ and $\left(X \cap B_{p}\right) N / N$. In particular $X=$ $\left(X \cap B_{p}\right) N$ is subnormal in $G$. Hence $X=N$ and so we conclude that $N=(N \cap A)(N \cap B)=N \cap B \leq B$, a contradiction. Therefore $B=X \cap B_{p}$. Thus $B$ is a $p$-group and furthermore we have $B N=$ $X=\left(X \cap A_{p}\right) B$, which implies that $X \cap A_{p} \neq 1$.

(2.2) Let $T$ be a normal $p^{\prime}$-subgroup of $A$. If $T\left(X \cap A_{p}\right)<A$, then $\left[T, X \cap A_{p}\right]=1$.

We set $S=T\left(X \cap A_{p}\right) N \leq G$. Since $\left(X \cap A_{p}\right) N=X=\left(X \cap A_{p}\right) B$, it is clear that $S=T\left(X \cap A_{p}\right) B$. If $T\left(X \cap A_{p}\right)<A$, then $S<G=N A$ and so $S / N=\left(T\left(X \cap A_{p}\right) N / N\right)(B N / N)$ is an $\mathcal{N}$-connected product by (3). In particular, $B N$ is subnormal in $S$ and so $B N \leq F(S)$. By (2.1) it follows that $X \cap A_{p} \leq B N \leq F(S)$. Therefore $\left[T, X \cap A_{p}\right] \leq$ $F(S) \cap O_{p^{\prime}}(A)=1$.

(2.3) $A=F(A) A_{p}, X \cap A_{p}=A_{p}$ and $N B=N A_{p}$.

Since $A \cong G / N$, we have that $F(A)$ is a $p^{\prime}$-group. Assume that $F(A)\left(X \cap A_{p}\right)<A$. Hence $\left[F(A), X \cap A_{p}\right]=1$ by $(2.2)$. Then it follows that $X \cap A_{p} \leq C_{A}(F(A)) \leq F(A)$, and so $X \cap A_{p}=1$, a contradiction. Therefore $F(A)\left(X \cap A_{p}\right)=A$. We conclude that $A_{p}=X \cap A_{p}$ and $A=F(A) A_{p}$. By (2.1) we have $N B=X=A_{p} B$. Since $A_{p} B$ is a Sylow $p$-subgroup of $G$ and $X \leq N A_{p}$, it follows that $N B=N A_{p}$.

(2.4) $F(A)$ is a $q$-group for some prime $q \neq p$. Moreover, $F(A) / \Phi(A)$ is a minimal normal subgroup of $A / \Phi(A)$ and $\Phi(F(A))=\Phi(A)=Z(A)=$ $C_{F(A)}\left(A_{p}\right)$.

As we have seen in (2.3), $F(A)$ is a $p^{\prime}$-group. Let $q$ be a prime divisor of $|F(A)|$. If $O_{q}(A) A_{p}<A$, then $\left[O_{q}(A), A_{p}\right]=1$ by $(2.2)$ and (2.3). Since $A_{p} \neq 1$, it is clear that $A_{p}$ is not centralized by $F(A)$. Consequently, there exists a prime $q \neq p$ such that $O_{q}(A) A_{p}=A$, which implies that $F(A)=O_{q}(A)$.

Notice that $F(A) / \Phi(A)=\operatorname{Soc}(A / \Phi(A))=L_{1} / \Phi(A) \times \cdots \times L_{s} / \Phi(A)$, where $L_{i} / \Phi(A)$ is a minimal normal subgroup of $A / \Phi(A)$ for $i=$ $1, \ldots, s$. If $s>1$, then $L_{i}<F(A)$ and $L_{i} A_{p}<A$ for $i=1, \ldots, s$. By (2.2) again we obtain that $A_{p}$ is centralized by $L_{1} \ldots L_{s}=F(A)$, 
a contradiction. Therefore $s=1$, which means that $F(A) / \Phi(A)$ is a minimal normal subgroup of $A / \Phi(A)$. We have

$$
A / \Phi(A)=(F(A) / \Phi(A))\left(A_{p} \Phi(A) / \Phi(A)\right)
$$

and obviously $A_{p} \neq A \neq A_{p} \Phi(A)$, so it follows that $A_{p} \Phi(A)$ is a nonnormal maximal subgroup of $A$. Thus, $O^{p^{\prime}}(A) \Phi(A)=A=O^{p^{\prime}}(A)$. Since $\Phi(A)<F(A)$, it follows from $(2.2)$ that $A_{p} \leq C_{A}(\Phi(A))$. Hence, $A=O^{p^{\prime}}(A) \leq C_{A}(\Phi(A))$ and we have $\Phi(A) \leq Z(A)$.

Since $\Phi(A) \leq Z(A) \leq C_{F(A)}\left(A_{p}\right)<F(A)$ and $Z(A)$ and $C_{F(A)}\left(A_{p}\right)$ are normal subgroups of $F(A) A_{p}=A$, we conclude that $\Phi(A)=Z(A)=$ $C_{F(A)}\left(A_{p}\right)$ because $F(A) / \Phi(A)$ is a chief factor of $A$.

Since $F(A) / \Phi(F(A))$ is a completely reducible $A_{p}$-module over $\operatorname{GF}(q)$ and $\Phi(F(A)) \leq \Phi(A) \leq F(A)$, there exists an $A_{p}$-module $T / \Phi(F(A))$ such that $F(A) / \Phi(F(A))=\Phi(A) / \Phi(F(A)) \oplus T / \Phi(F(A))$. Consequently, $A=F(A) A_{p}=\Phi(A) T A_{p}=T A_{p}$. Then $F(A)=T$ and so we have that $\Phi(A)=\Phi(F(A))$.

(2.5) Let $\alpha \in A, a \in A_{p}$. Then $N, \alpha, a$ satisfy Condition (*) of Lemma 8.

By (2.3) $N B=N A_{p}$, so we have $a=\mu b$ for some $\mu \in N, b \in B$. We recall that $N, \alpha, b$ satisfy Condition $(*)$ of Lemma 8 . Since $\langle\alpha, \mu b\rangle=$ $\langle\alpha, a\rangle \leq A \in \mathcal{N}^{2}$, we conclude by Lemma 8 (4) that $N, \alpha, a$ satisfy Condition $(*)$.

(2.6) $F(A)$ has exponent $q$ and $\Phi(A)$ has order 1 or $q$.

Let $x \in F(A) \backslash \Phi(A)$. First we claim that $C_{N}(x) \neq 1$. If $C_{N}(x)=1$, then $[N, x]=N$ by coprime action. In particular, $C_{N}\left(\langle x, a\rangle^{\mathcal{N}}\right)[N, x]=$ $N$ for all $a \in A_{p}$. By (2.5) $N, x, a$ satisfy Condition (*) of Lemma 8, so it follows from Lemma 8 (3) and (6) that $N=C_{N}\left(\langle x, a\rangle^{\mathcal{N}}\right)$. Thus, $\langle x, a\rangle^{\mathcal{N}} \leq N \cap A=1$ and we have $[x, a]=1$. This holds for all $a \in A_{p}$, which means that $x \in C_{F(A)}\left(A_{p}\right)=\Phi(A)$, a contradiction. Therefore $C_{N}(x) \neq 1$.

Since $F(A)$ is a $q$-group, we have that $x^{q} \in \Phi(F(A))=Z(A)$ by (2.4). Now it is clear that $C_{N}\left(x^{q}\right)$ is a normal subgroup of $N A=G$. Since $1 \neq C_{N}(x) \leq C_{N}\left(x^{q}\right)$, it follows that $C_{N}\left(x^{q}\right)=N$ and hence that $x^{q} \in N \cap A=1$. This proves $x^{q}=1$ for all $x \in F(A) \backslash \Phi(A)$.

Now, let $z \in \Phi(A)$. We can consider $y \in F(A) \backslash \Phi(A)$, then $y z \in$ $F(A) \backslash \Phi(A)$. Since $\Phi(A)=Z(A)$, we have that $1=(y z)^{q}=y^{q} z^{q}=z^{q}$. Therefore $x^{q}=1$ for all $x \in F(A)$.

Since $N$ is an irreducible and faithful $A$-module over $\operatorname{GF}(p)$, it follows that $Z(A)$ is cyclic (see [8, Corollary B.9.4]). Now the result is clear. 
(2.7) Let $x \in F(A), a \in A_{p}$. Then $\left[x^{a}, x\right] \in\langle x, a\rangle^{\mathcal{N}}=\left\langle a^{x}, a\right\rangle^{\mathcal{N}}$.

We set $z=\left[x^{a}, x\right]$. Notice that $z \in F(A)^{\prime} \leq \Phi(A)=Z(A)$. We have $z=\left(x^{a}\right)^{-1} x^{-1} x^{a} x$ and so $x^{a} z x^{-1}=[x, a]$. Thus, $[x, a]=x^{a} x^{-1} z=$ $\left[a, x^{-1}\right] z$. In particular, since $x$ and $a$ have coprime orders, we have that $z \in[\langle x\rangle,\langle a\rangle]=\langle x, a\rangle^{\mathcal{N}}$. Since $[x, a]=a^{-1} x a x^{-1} z$, we have that $\left\langle a^{x}, a\right\rangle=\left\langle a^{-1} a^{x}, a\right\rangle=\langle[x, a], a\rangle=\left\langle x a x^{-1} z, a\right\rangle=\left\langle a^{x^{-1}} z, a\right\rangle$. Since $z \in Z(A)$ and $a^{x^{-1}}$ and $z$ have coprime orders, it follows that $\left\langle a^{x}, a\right\rangle=\left\langle a^{x^{-1}}, z, a\right\rangle$. It is clear that this subgroup is normalized by $x$ and so it is a normal subgroup of $\langle x, a\rangle$. Let $Q$ denote the normal closure of $\langle x\rangle$ in $\langle x, a\rangle$. Since $Q \leq F(A)$ and $\langle x, a\rangle=\left\langle a^{x}, a\right\rangle Q$, it follows that $\langle x, a\rangle^{\mathcal{N}}=\left\langle a^{x}, a\right\rangle^{\mathcal{N}}$.

(2.8) Assume that $F(A)$ is not abelian. Then $F(A)$ is an extraspecial $q$ group. Moreover, there exist $x \in F(A) \backslash \Phi(A), a \in A_{p}$ such that $\Phi(A)=\left\langle\left[x^{a}, x\right]\right\rangle$.

Assume that $F(A)$ is not abelian. Then $1 \neq \Phi(F(A))=\Phi(A)$ by $(2.4)$ and it follows that $\Phi(F(A))$ has order $q$ by (2.6). Therefore, $F(A)^{\prime}=$ $\Phi(F(A))$. Furthermore, we have that $\Phi(A)=Z(A) \leq Z(F(A))<$ $F(A)$, whence $Z(F(A))=\Phi(A)$. Thus we have that $\Phi(F(A))$ has order $q$ and $Z(F(A))=\Phi(F(A))=F(A)^{\prime}$, i. e., $F(A)$ is extraspecial. We can consider some $x \in F(A) \backslash \Phi(A)$. Since $\Phi(A)\langle x\rangle\left[\langle x\rangle, A_{p}\right]$ is a normal subgroup of $A$ contained in $F(A)$ and $x \notin \Phi(A)$, we deduce that $\Phi(A)\langle x\rangle\left[\langle x\rangle, A_{p}\right]=F(A)$. Thus, we have that $\langle x\rangle\left[\langle x\rangle, A_{p}\right]=$ $F(A)$. If $x^{a} \in C_{G}(x)$ for all $a \in A_{p}$, then $F(A) \leq C_{G}(x)$ and $x \in$ $Z(F(A))=\Phi(A)$, a contradiction. Therefore, there exists $a \in A_{p}$ such that $\left[x^{a}, x\right] \neq 1$. Since $\left[x^{a}, x\right] \in F(A)^{\prime}=\Phi(A)$, the desired conclusion follows.

(2.9) Assume that $F(A)$ is not abelian and let $x \in F(A) \backslash \Phi(A)$. Then $N$, regarded as a $\mathrm{GF}(p)\langle x\rangle$-module, is a direct sum of regular $\mathrm{GF}(p)\langle x\rangle$ modules. In particular, we have that $|N|=\left|C_{N}(x)\right|^{q}$.

Assume that $F(A)$ is not abelian. Notice that $N$ is an irreducible $A$ module. Let $V$ be an irreducible $F(A)$-submodule of $N$. By Clifford's theorem, we have that $N=V^{a_{1}} \oplus \cdots \oplus V^{a_{s}}$ for certain $a_{i} \in A_{p}$ and $V^{a_{i}}$ is an irreducible $F(A)$-module for $i=1, \ldots, s$.

Let us see first that $V^{a_{i}}$ is a faithful $F(A)$-module for $i=1, \ldots, s$. Notice that $C_{F(A)}\left(V^{a_{i}}\right)=C_{F(A)}(V)^{a_{i}}$ is a normal subgroup of $F(A)$ for $i=1, \ldots, s$. Suppose that $C_{F(A)}(V) \neq 1$. By $(2.8), Z(F(A))$ has order $q$, so it follows that $Z(F(A)) \leq C_{F(A)}(V)$ and so we have that $Z(F(A)) \leq C_{F(A)}\left(V^{a_{i}}\right)$ for $i=1, \ldots, s$. Thus, $Z\left(F(A) \leq C_{F(A)}(N)=1\right.$, a contradiction. Therefore $C_{F(A)}(V)=1$ and $C_{F(A)}\left(V^{a_{i}}\right)=1$ for $i=1, \ldots, s$. 
Now $V^{a_{i}}$ is an irreducible and faithful $F(A)$-module for $i=1, \ldots, s$. Let $x \in F(A) \backslash \Phi(A)$. Then, $x \in F(A) \backslash \Phi(F(A))$ and $x$ has order $q$. By $(2.8), F(A)$ is an extraspecial $q$-group, so we can apply [8, Corollary B.9.20] to deduce that $V^{a_{i}}$ is a direct sum of regular $\mathrm{GF}(p)\langle x\rangle$-modules. Therefore $N$ is a direct sum of regular $\operatorname{GF}(p)\langle x\rangle$-modules. In other words, we have that $N=L_{1} \times \cdots \times L_{t}$, with $L_{i}$ normalized by $x$, $\left|L_{i}\right|=p^{q}$ and $\left|C_{L_{i}}(x)\right|=p$ for $i=1, \ldots, t$. Now it is straightforward to verify that $C_{N}(x)=C_{L_{1}}(x) \times \cdots \times C_{L_{t}}(x)$, so we can conclude that $|N|=\left(p^{q}\right)^{t}=\left|C_{N}(x)\right|^{q}$.

(2.10) $F(A)$ is an elementary abelian $q$-group and $\Phi(A)=1$.

Suppose that $F(A)$ is not abelian. By (2.8), there exist $x \in F(A) \backslash$ $\Phi(A), a \in A_{p}$ such that $\Phi(A)=\left\langle\left[x^{a}, x\right]\right\rangle$. Notice that $N, x, a$ and also $N, a^{x}, a$ satisfy Condition (*) of Lemma 8 by (2.5). By (2.7) we have that $\Phi(A) \leq\langle x, a\rangle^{\mathcal{N}}=\left\langle a^{x}, a\right\rangle^{\mathcal{N}}$. We let $C=C_{N}\left(\langle x, a\rangle^{\mathcal{N}}\right)=$ $C_{N}\left(\left\langle a a^{x}, a\right\rangle^{\mathcal{N}}\right)$ and $R=\left[N,\langle x, a\rangle^{\mathcal{N}}\right]=\left[N,\left\langle a a^{x}, a\right\rangle^{\mathcal{N}}\right]$. We have that $\Phi(A)=\Phi(F(A)) \neq 1, C_{N}(\Phi(A))<N$ and $C_{N}(\Phi(A))$ is a normal subgroup of $G$, so it follows that $C_{N}(\Phi(A))=1$. Therefore $C=1$ and so, by Lemma 8 (1) and (2), we conclude that $R=N$ and $C_{N}(x) \times$ $C_{N}(a)=N=C_{N}\left(a^{x}\right) \times C_{N}(a)$. Hence $\left|C_{N}(x)\right|=\left|C_{N}\left(a^{x}\right)\right|=\left|C_{N}(a)\right|$, and consequently $|N|=\left|C_{N}(x)\right|^{2}$. On the other hand, we have $|N|=$ $\left|C_{N}(x)\right|^{q}$ by (2.9), and so $q=2$. Since $F(A)$ has exponent $q$, it follows that $F(A)$ is abelian, a contradiction. Therefore $F(A)$ is abelian and it is an elementary abelian $q$-group. Moreover, we have that $\Phi(A)=$ $\Phi(F(A))=1$.

(2.11) Let $a \in Z\left(A_{p}\right),|\langle a\rangle|=p$. If $S \leq F(A)$ and $|S|=q$, then $S^{a} \neq S$. In particular, $p \neq 2$.

Let $S \leq F(A)$ and $|S|=q$. Then $S=\langle x\rangle$ with $1 \neq x \in F(A)$. We suppose that $S^{a}=S$ and obtain a contradiction. Notice that $N, x, a$ satisfy Condition $(*)$ of Lemma 8 by $(2.5)$. We let $C=C_{N}\left(\langle x, a\rangle^{\mathcal{N}}\right)$ and $N_{1}=C[N,\langle x\rangle]=C[N, S]$. Then $a$ normalizes $N_{1}$ and from Lemma 8 (6) it follows that $N=C$. Thus, $\langle x, a\rangle^{\mathcal{N}} \leq N \cap A=1$. Since $x$ and $a$ have coprime orders, we conclude that $x \in C_{F(A)}(a)$. By (2.4) and (2.10), $F(A)$ is a minimal normal subgroup of $A$. Since $1 \neq C_{F(A)}(a)$ and $C_{F(A)}(a)$ is normalized by $F(A) A_{p}=A$, it follows that $C_{F(A)}(a)=F(A)$ whence $a \in F(A)$, a contradiction. This proves that $S^{a} \neq S$.

Let us see that $p \neq 2$. We can take $1 \neq x \in F(A)$. If $p=2$, then $x x^{a}$ is fixed under $a$ since $F(A)$ is abelian and $a^{2}=1$. Hence $x x^{a}=1$ and so $\langle x\rangle^{a}=\langle x\rangle$, a contradiction. Therefore $p \neq 2$. 
(2.12) The final contradiction.

Since $Z\left(A_{p}\right) \neq 1$, we can take $a \in Z\left(A_{p}\right)$ such that $|\langle a\rangle|=p$. We also take $1 \neq x \in F(A)$. By $(2.11),\langle x\rangle^{a} \neq\langle x\rangle$. In particular $x^{a} \neq x$, $\langle x, a\rangle^{\mathcal{N}} \neq 1$ and $C_{N}\left(\langle x, a\rangle^{\mathcal{N}}\right) \neq N$. Notice that by (2.5) both $N, x, a$ and $N, a^{x}, a$ satisfy Condition (*) of Lemma 8. Using (2.7) we set

$$
C=C_{N}\left(\langle x, a\rangle^{\mathcal{N}}\right)=C_{N}\left(\left\langle a^{x}, a\right\rangle^{\mathcal{N}}\right), R=\left[N,\langle x, a\rangle^{\mathcal{N}}\right]=\left[N,\left\langle a a^{x}, a\right\rangle^{\mathcal{N}}\right] .
$$

Since $C \neq N$, it follows from Lemma 8 that $R \neq 1$ and $C \cap R=1$. Let $Q$ denote the normal closure of $\langle x\rangle$ in $\langle x, a\rangle$. Since $Q \leq F(A)$, $Q$ is an elementary abelian $q$-group. We have that $\langle x, a\rangle=Q\langle a\rangle$ and $\langle x, a\rangle^{\mathcal{N}}=\left\langle a^{x}, a\right\rangle^{\mathcal{N}} \leq Q$.

Since $R$ is normalized by $\langle x, a\rangle$ and $R \neq 1$, we can consider an irreducible $\operatorname{GF}(p) Q$-submodule $V$ of $R$. Because $Q$ is abelian, $Q / C_{Q}(V)$ is abelian, which implies that $Q / C_{Q}(V)$ is cyclic (see [8, Proposition B.9.3]). We have that $C \cap R=1$, and so $V$ is not centralized by $\langle x, a\rangle^{\mathcal{N}}$. In particular, $C_{Q}(V)<Q$ and therefore $Q / C_{Q}(V)$ has order $q$. Observe that $Q$ is completely reducible as $\operatorname{GF}(q)\langle a\rangle$-module. We claim that $C_{Q}(V)^{a} \neq C_{Q}(V)$. Otherwise, $C_{Q}(V)$ is a $\operatorname{GF}(q)\langle a\rangle$-submodule of $Q$. Then we have $Q=C_{Q}(V) \oplus S$ for some $\operatorname{GF}(q)\langle a\rangle$-submodule $S$ of $Q$. Therefore $S$ has order $q, S \leq F(A)$ and $S^{a}=S$, which contradicts (2.11). This proves that $C_{Q}(V)^{a} \neq C_{Q}(V)$.

It is clear that $V, V^{a}, V^{a^{2}}, \ldots, V^{a^{p-1}}$ are irreducible $Q$-submodules of $R$. Let us see that their sum is direct. Since the sum of these submodules is a completely reducible $Q$-module, it is sufficient to show that $V^{a^{i}}$ and $V^{a^{j}}$ are not isomorphic as $Q$-modules if $a^{i} \neq a^{j}$. If this is not so, then for some $a^{i} \neq a^{j}$ we have that $C_{Q}\left(V^{a^{i}}\right)=C_{Q}\left(V^{a^{j}}\right)$ and consequently, $C_{Q}(V)^{a^{i}}=C_{Q}(V)^{a^{j}}$. Since $\langle a\rangle$ has order $p$, it follows that $C_{Q}(V)^{a}=$ $C_{Q}(V)$, a contradiction. Therefore we may write $L=V \oplus V^{a} \oplus \cdots \oplus V^{a^{p-1}}$. It is clear that $L$ is an $\langle x, a\rangle$-submodule of $R$.

We notice that $L$ is isomorphic to $V^{Q\langle a\rangle}$, the induced module of $V$ from $Q$ to $Q\langle a\rangle=\langle x, a\rangle$. Then, by Mackey's theorem, we obtain that $L_{\langle a\rangle} \cong\left(V^{Q\langle a\rangle}\right)_{\langle a\rangle} \cong\left(V_{Q \cap\langle a\rangle}\right)^{\langle a\rangle} \cong\left(V_{\{1\}}\right)^{\langle a\rangle} \cong \mathrm{GF}(p)\langle a\rangle \oplus \cdots \oplus \operatorname{GF}(p)\langle a\rangle$, a direct sum of copies of the regular $\operatorname{GF}(p)\langle a\rangle$-module: $L=L_{1} \oplus \cdots \oplus L_{s}$. It is straightforward to verify that $C_{L}(a)=C_{L_{1}}(a) \oplus \cdots \oplus C_{L_{s}}(a)$, so we can conclude that $\left|C_{L}(a)\right|=p^{s}$. Hence, $|L|=p^{p s}=\left|C_{L}(a)\right|^{p}$.

On the other hand, recall that $N, a^{x}$, $a$ satisfy Condition(*) of Lemma 8. Since $\left\langle a^{x}, a\right\rangle^{\mathcal{N}} \leq Q$, we have that $O_{p}\left(\left\langle a^{x}, a\right\rangle^{\mathcal{N}}\right)=1$. Moreover, $L$ is 
normalized by $\langle x, a\rangle$, so it follows that $L, a^{x}, a$ satisfy Condition $(*)$ of Lemma 8. Observe that $C_{L}\left(\left\langle a^{x}, a\right\rangle^{\mathcal{N}}\right)=C \cap L \leq C \cap R=1$. Therefore, it follows from Lemma $8(2)$ that $L=C_{L}\left(a^{x}\right) \times C_{L}(a)$. Consequently, $|L|=\left|C_{L}\left(a^{x}\right)\right|\left|C_{L}(a)\right|=\left|C_{L}(a)\right|^{2}$. Finally, this implies that $p=2$, which contradicts (2.11).

Remark 1 In contrast to all essential properties of $\mathcal{N}$-connectedness(cf. [12]), Theorem 1 does not generalize in the obvious way from products with two factors to products with $n \geq 3$ factors. It is easy to construct examples of groups $G=A B C$ of pairwise permuting subgroups $A, B, C$ such that $A, B$ and $A, C$ and $B, C$ are $\mathcal{N}^{2}$-connected, but $A F(G) / F(G)$ and $B F(G) / F(G)$ are not $\mathcal{N}$-connected; e. g. $G=\operatorname{Sym}(4), A=\langle(12)\rangle, B=\langle(123)\rangle, C=$ $\langle(12)(34)\rangle \times\langle(13)(24)\rangle$.

However, let us consider another generalizing condition to the case $G=$ $S_{1} \cdots S_{n}$ where $G$ is soluble with $S_{1}, \ldots, S_{n}$ pairwise permuting subgroups. It follows from [12, Proposition 1 (5)] that, in this case, the subgroups $S_{1}, \ldots, S_{n}$ are pairwise $\mathcal{N}$-connected if and only if $\left\langle a_{1}, \ldots, a_{n}\right\rangle \in \mathcal{N}$ for all $a_{i} \in S_{i}, i=1, \ldots, n$. Now, assume that $G=S_{1} \cdots S_{n}$ is a product of pairwise permuting subgroups such that $\left\langle a_{1}, \ldots, a_{n}\right\rangle \in \mathcal{N}^{2}$ for all $a_{i} \in S_{i}$, $i=1, \ldots, n$. Then it is clear that, for every $i=1, \ldots, n, S_{i}$ and $\prod_{j \neq i} S_{j}$ are $\mathcal{N}^{2}$-connected subgroups of $G$. By Theorem 1 it follows that the subgroups $S_{1} F(G) / F(G), \ldots, S_{n} F(G) / F(G)$ are pairwise $\mathcal{N}$-connected and so that $\left\langle a_{1}, \ldots, a_{n}\right\rangle F(G) / F(G) \in \mathcal{N}$ for all $a_{i} \in S_{i}, i=1, \ldots, n$.

It is still possible to state another generalization for products of more than two factors. Let the soluble group $G=S_{1} \cdots S_{n}$ be again a product of pairwise permuting subgroups with $n \geq 3$. Assume that $S_{1}$ and $S_{2}$ are $\mathcal{N}^{2}$-connected and $S_{i}$ and $S_{j}$ are $\mathcal{N}$-connected for all $i \neq j$ with $\{i, j\} \neq$ $\{1,2\}$. For any prime $p$, let $P \in \operatorname{Syl}_{p}\left(S_{1}\right)$ and $Q \in \operatorname{Syl}_{p}\left(S_{2}\right)$ such that $P Q \in$ $\operatorname{Syl}_{p}\left(S_{1} S_{2}\right)$. From [12, Proposition $\left.1(3)\right]$ and Lemma 6 we deduce that $P Q$ and $S_{3} \cdots S_{n}$ are $\mathcal{N}$-connected permuting subgroups. Consequently, $O_{p}\left(S_{1} S_{2}\right) \unlhd P Q \unlhd \unlhd P Q S_{3} \cdots S_{n}$ and so also $O_{p}\left(S_{1} S_{2}\right) \unlhd \unlhd S_{1} S_{2} S_{3} \cdots S_{n}$. It follows now from Theorem 1 that $\left\langle a_{1}, a_{2}\right\rangle^{\mathcal{N}} \leq F\left(S_{1} S_{2}\right) \leq F\left(S_{1} \cdots S_{n}\right)=$ $F(G)$ for all $a_{1} \in S_{1}$ and $a_{2} \in S_{2}$, that is, $S_{1} F(G) / F(G)$ and $S_{2} F(G) / F(G)$ are $\mathcal{N}$-connected.

Remark 2 The hypothesis in Theorem 1 that $G$ is the product of the $\mathcal{N}^{2}$-connected subgroups $A$ and $B$ is essential; it cannot be replaced by $G=\langle A, B\rangle$. For instance, let $G=(\operatorname{Alt}(4) \times \operatorname{Alt}(4)) C_{2}$ be the wreath product of Alt(4) with $C_{2}, A=\operatorname{Alt}(4) \times 1, B=C_{2}$. Then $G=\langle A, B\rangle$, $A$ and $B$ are $\mathcal{N}^{2}$-connected, but $A F(G) / F(G)$ and $B F(G) / F(G)$ are not $\mathcal{N}$-connected. 
We draw some immediate consequences of Theorem 1.

Corollary 1 Let $\mathcal{F}$ be a class of soluble groups and assume that

(i) $\mathcal{F}$ is a Q-closed Fitting class, or

(ii) $\mathcal{F}$ is a formation containing $\mathcal{N}$.

If the group $G=A B$ is the $\mathcal{N}^{2}$-connected product of the subgroups $A$ and $B$, then $A, B \in \mathcal{F}$ implies $G \in \mathcal{N} \mathcal{F}$, and $G \in \mathcal{N} \mathcal{F}$ implies $A, B \in \mathcal{N} \mathcal{F}$.

Proof. Suppose that $A, B \in \mathcal{F}$. Then $G=A B$ is an $\mathcal{S}$-connected product of the soluble subgroups $A$ and $B$. Hence $G$ is a soluble group by [6, Theorem]. By Theorem 1 and Q-closure of $\mathcal{F}, G / F(G)$ is the $\mathcal{N}$-connected product of the $\mathcal{F}$-subgroups $A F(G) / F(G)$ and $B F(G) / F(G)$. Each of the conditions $(i)$ and $(i i)$ implies that $\mathcal{N}$-connected products of $\mathcal{F}$-subgroups are $\mathcal{F}$-subgroups, by Lemma 2 (1) and [12, Proposition 3] respectively. Therefore, $G \in \mathcal{N} \mathcal{F}$. The second part is proved similarly.

As a particular case of Corollary 1 we state explicitly:

Corollary 2 If the group $G=A B$ is the $\mathcal{N}^{2}$-connected product of the soluble subgroups $A$ and $B$ of nilpotent length at most $l$, then $G$ is soluble of nilpotent length at most $l+1$.

\section{Concluding remarks}

It is natural to ask whether Theorem 1 can be extended to the general case of $\mathcal{N} \mathcal{F}$-connected products, $\mathcal{F}$ a formation. The following example shows that this is only possible for formations $\mathcal{F}$ containing all finite abelian groups.

Example Let $\mathcal{F}$ be a formation such that $\mathcal{A} \nsubseteq \mathcal{F}$. Then there is a cyclic group $C=\langle c\rangle$ which is not contained in $\mathcal{F}$. We consider now $V$ a faithful $C$ module over $\operatorname{GF}(p)$, for a prime $p \notin \sigma(C)$, and $G=[V] C$ the corresponding semidirect product.

Obviously

$$
\langle g\rangle \in \mathcal{N} \subseteq \mathcal{N} \mathcal{F} \text { for all } g \in G,
$$

this is to say that $G=G B$ is the $\mathcal{N} \mathcal{F}$-connected product of $G$ and $B=1$. But

$$
\langle c\rangle^{\mathcal{F}} \not \leq F(G)=V,
$$

that is, $G / F(G)$ and $B F(G) / F(G)$ are not $\mathcal{F}$-connected. 
We have not been able yet to prove a version of Theorem 1 for any soluble $\mathcal{N} \mathcal{F}$-connected product $G=A B$, where $\mathcal{F} \supseteq \mathcal{A}$ is a formation. This is however possible under certain conditions on the factors $A$ and $B$. In the following we just present one such result which has already interesting consequences (Corollaries 3 and 4).

Proposition 1 Let $\mathcal{F}$ be a formation of soluble groups containing all abelian groups. Let $G$ be a soluble group such that $G=A B$ is the $\mathcal{N} \mathcal{F}$-connected product of the subgroups $A$ and $B$. Assume that one of the factors $A, B$ is normally embedded in $G$. Then

$$
G / F(G)=(A F(G) / F(G))(B F(G) / F(G))
$$

is an $\mathcal{F}$-connected product of the two factors.

Proof. We observe first that the statement of the theorem is equivalent to the fact that $\langle a, b\rangle^{\mathcal{F}} \leq F(G)$ for all $a \in A$ and all $b \in B$.

Assume that the result is false and let $G$ be a counterexample with $|G|$ minimal. By Lemma 9 we have that $G$ has a unique minimal normal subgroup $N, N \not \leq A$ and $N \not \leq B$. In particular, for the prime $p$ dividing $|N|$, it follows that $p|| A \mid$ and $p|| B \mid$. We may assume that $A$ is normally embedded in $G$. Therefore we have $1 \neq A_{p} \in \operatorname{Syl}_{p}(A)$ and $A_{p} \in \operatorname{Syl}_{p}(K)$ for some normal subgroup $K$ of $G$. Since $N$ is the unique minimal normal subgroup of $G$, we have $N \leq K$ and so $N \leq A_{p}$, a contradiction which concludes the proof.

For a group $G$ we set $F_{0}(G)=1$ and $F_{k}(G)=F\left(G \bmod F_{k-1}(G)\right)$ for $k \geq 1$.

Corollary 3 Let $G$ be a soluble group, $g \in G$ and $k \geq 1$. Then $\langle g, h\rangle \in \mathcal{N}^{k}$ for all $h \in G$ if and only if $g \in Z_{\infty}\left(G \bmod F_{k-1}(G)\right)$.

Proof. If $g \in Z_{\infty}\left(G \bmod F_{k-1}(G)\right)$, then $\langle g, h\rangle F_{k-1}(G) / F_{k-1}(G)$ is nilpotent and so $\langle g, h\rangle \in \mathcal{N}^{k}$ for all $h \in G$. We will show the other implication by induction on $k$.

If $k=1$, then $\langle g\rangle$ and $G$ are $\mathcal{N}$-connected and $g \in Z_{\infty}(G)$ by Lemma $2(2)$. Suppose inductively that the result holds for $k \geq 1$. Assume that $\langle g, h\rangle \in$ $\mathcal{N}^{k+1}$ for all $h \in G$. Then $\langle g\rangle$ and $G$ are $\mathcal{N}^{k+1}$-connected. It follows from Proposition 1 with $\mathcal{F}=\mathcal{N}^{k}$ that $\langle\bar{g}\rangle$ and $\bar{G}$ are $\mathcal{N}^{k}$-connected, where we denote by bars the images in the factor group $G / F(G)$. By inductive hypothesis we have $\bar{g} \in Z_{\infty}\left(\bar{G} \bmod F_{k-1}(\bar{G})\right)$. Since the $\mathcal{N}^{k-1}$-radical of $\bar{G}$ is $F_{k-1}(G / F(G))=F_{k}(G) / F(G)$, we conclude that $g \in Z_{\infty}\left(G \bmod F_{k}(G)\right)$. This completes the induction argument. 
Corollary 4 For a soluble group $G$, an element $g \in G$ and $k \geq 1$, the following statements are equivalent:

1. $g \in F_{k}(\langle g, h\rangle)$ for all $h \in G$.

2. $\langle g, h\rangle \in \mathcal{N}^{k}$ for all $h \in F_{k}(G)$ and $\langle g, h\rangle \in \mathcal{N}^{k+1}$ for all $h \in G$.

3. $g \in F_{k}(G)$.

Proof. Assume that Condition 1 holds. Then it is clear that $\langle g, h\rangle \in \mathcal{N}^{k+1}$ for all $h \in G$. On the other hand, if $h \in F_{k}(G)$ we deduce that $\langle g, h\rangle \leq$ $F_{k}(\langle g, h\rangle)$ and so $\langle g, h\rangle \in \mathcal{N}^{k}$. Hence Condition 1 implies Condition 2.

Assume now that Condition 2 holds and we prove Condition 3. First it follows from Corollary 3 that $g \in F_{k+1}(G)$ since $\langle g, h\rangle \in \mathcal{N}^{k+1}$ for all $h \in G$. Now if $x=a s \in X:=F_{k}(G)\langle g\rangle$ with $a \in F_{k}(G)$ and $s \in\langle g\rangle$, then $\langle x, g\rangle \leq\langle a, g\rangle \in \mathcal{N}^{k}$ by hypothesis. We deduce that $g \in F_{k}(X)=F_{k}(G)$ by Corollary 3 again and since $X \unlhd \unlhd G$. This proves Condition 3 .

Finally Condition 1 is easily deduced from Condition 3.

Remark 3 The equivalence of Condition 1 and Condition 3 in Corollary 4 is a result of Flavell [10, Theorem 2.1] in a paper that was motivated by the following conjecture of the same author: "The soluble radical of a finite group $G$ coincides with the set of elements $y \in G$ satisfying that $\langle y, x\rangle$ is soluble for all $x \in G^{\prime \prime}$. This conjecture has been recently proven in [11].

\section{References}

[1] Amberg, B., Franciosi, S. and De Giovanni, F.: Products of Groups. The Clarendon Press, Oxford Univ. Press, New York, 1992.

[2] Ballester-Bolinches, A. And Pedraza-Aguilera, M. C.: On finite soluble products of $\mathcal{N}$-connected groups. J. Group Theory 2 (1999), no. 3, 291-299.

[3] Beidleman, J. and Heineken, H.: Pairwise $\mathcal{N}$-connected products of certain classes of finite groups. Comm. Algebra 32 (2004), no. 12, 4741-4752.

[4] Brandl, R.: Zur Theorie der untergruppenabgeschlossenen Formationen: Endliche Varietäten. J. Algebra 73 (1981), no. 1, 1-22.

[5] Carocca, A.: A note on the product of $\mathcal{F}$-subgroups in a finite group. Proc. Edinburgh Math. Soc. (2) 39 (1996), no. 1, 37-42.

[6] Carocca, A.: Solvability of factorized finite groups. Glasg. Math. J. 42 (2000), no. 2, 271-274.

[7] Carter, R. W., Fischer, B. and Hawkes, T. O.: Extreme classes of finite soluble groups. J. Algebra 9 (1968), 285-313. 
[8] Doerk, K. And Hawkes, T.: Finite soluble groups. De Gruyter Expositions in Mathematics 4. Walter de Gruyter, Berlin, 1992

[9] Flavell, P.: Finite groups in which every two elements generate a soluble subgroup. Invent. Math. 121 (1995), no. 2, 279-285.

[10] Flavell, P.: A characterization of $F_{2}(G)$. J. Algebra 255 (2002), no. 2, 271-287.

[11] Guralnick, R., Kunyavskil, B., Plotkin, E. And Shalev, A.: Thompson-like characterizations of the solvable radical. J. Algebra 300 (2006), no. 1, 363-375.

[12] Hauck, P., Martínez-Pastor, A. and Pérez-Ramos, M. D.: Products of $\mathcal{N}$-connected groups. Illinois J. Math. 47 (2003), no. 4, 1033-1045.

[13] Lennox, J. C. And Stonehewer, S. E.: Subnormal subgroups of groups. Oxford Mathematical Monographs. Oxford Science Publications. The Clarendon Press Oxford University Press, New York, 1987.

[14] MAier, R.: A completeness property of certain formations. Bull. London Math. Soc. 24 (1992), no. 6, 540-544.

[15] Thompson, J. G.: Non-solvable groups all of whose local subgroups are solvable, I-VI Bull. Amer. Math. Soc. 74 (1968), 383-437; Pacific J. Math. 33 (1970), 451-536; 39 (1971), 483-534; 48 (1973), 511-592; 50 (1974), 215-297; 51 (1974), 573-630.

Recibido: 8 de septiembre de 2006

M. Pilar Gállego

Departamento de Matemáticas

Universidad de Zaragoza

Edificio Matemáticas, Ciudad Universitaria. 50009 Zaragoza, Spain pgallego@unizar.es

Peter Hauck Wilhelm-Schickard-Institut für Informatik

Universität Tübingen

Sand 14. 72076 Tübingen, Germany hauck@informatik. uni-tuebingen.de

M. Dolores Pérez-Ramos Departament d'Àlgebra Universitat de València

C/ Doctor Moliner 50. 46100 Burjassot (València), Spain Dolores.Perez@uv.es

Research supported by Proyectos MTM2004-06067-C02-02 and MTM2007-68010-C03-03, Ministerio de Educación y Ciencia and FEDER, Spain. 